\title{
Thermal Management for Multi-Phase Current Mode Buck Converters
}

By

$\mathrm{Ke} \mathrm{Cao}$

A thesis submitted in conformity with the requirements for the degree of Master of Applied Science

Graduate Department of Electrical and Computer Engineering University of Toronto

(C) Copyright by Ke Cao 2011 


\title{
Thermal Management for Multi-Phase Current Mode Buck Converters
}

\author{
$\mathrm{Ke} \mathrm{Cao}$ \\ Master of Applied Science \\ Graduate Department of Electrical and Computer Engineering \\ University of Toronto
}

2011

\begin{abstract}
The main goal of this thesis is to develop an active thermal management control scheme for multi-phase current mode buck converters in order to improve the long term reliability of the converters. A thermal management unit (TMU) with independent linear compensators for the thermal loops is incorporated into the existing digital controller to regulate the current through each phase so that equal temperature distribution is achieved across all phases. A lumped parameter thermal model of the multi-phase converter is built as the basis of the TMU. MATLAB simulation results are used to verify the TMU concept. Experimental results from a digitally controlled $12 \mathrm{~V}$ to $1 \mathrm{~V}, 50 \mathrm{~A}, 250 \mathrm{kHz}$ four-phase peak current mode buck converter demonstrate the effectiveness of the proposed thermal management technique in the presence of uneven airflow. The steady-state performance, dynamic transient performance, effect of gate drive voltage and efficiency measurements are investigated and discussed.
\end{abstract}




\section{Acknowledgements}

I would like to express my sincere gratitude to my supervisors, Professor Wai Tung Ng and Professor Olivier Trescases, for their guidance and support throughout my studies at University of Toronto. Their expertise and vision in the area of DC-DC converters have provided me with great motivation and encouragement. They are also very caring, understanding and approachable mentors that support the students' best interest and look out for the students. Their high level of awareness, wisdom, vision, poise, charisma, drive, technical excellence, as well as their willingness to inspire the young generation has set them to be great role models. It is truly my honour to have the privilege to work with them.

This work would not have been possible without the help of my colleague Mohammad Shawkat Zaman, who has provided excellent technical support. I really appreciate his time, effort and dedication. I would like to thank my colleagues in my lab for creating a nice working environment, and their friendship along the way: Jing Wang, April Zhao, Andrew Shorten, Shuang Xie, Gang Xie, Junming Lee, and Stella Tang in my lab. I would also like to thank Shahab Poshtkouhi, Victor Wen, Mazhar Moshivrazivi, Ahsan Zaman for their support.

I should also thank all the researchers in the Electronics Group for creating an environment where knowledge sharing and crossing training are highly encouraged.

Finally, I would like to express my gratitude to Jaro Pristupa's CAD support and Edward Xu's providence on various lab equipments and tools. 


\section{Table of Contents}

Chapter 1 Thermal Variations in Multi-Phase Converters...................................................... 1

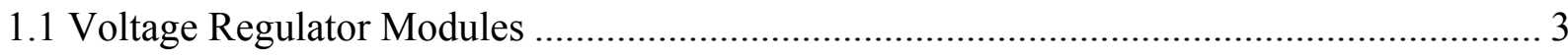

1.1.1 Single Phase versus Multi-Phase Converters ...................................................... 4

1.1.2 Average Current Mode Control vs. Peak-Current Programmed Mode ....................... 10

1.2 Thermal Management for Multi-Phase Converters ................................................ 12

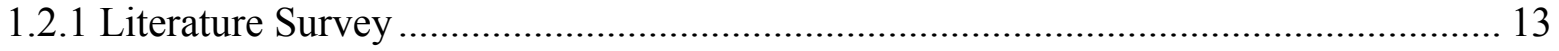

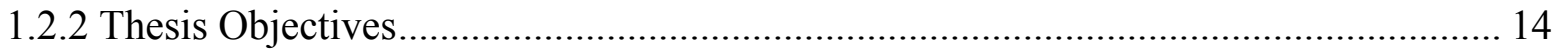

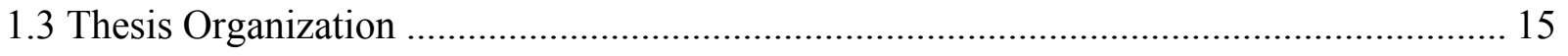

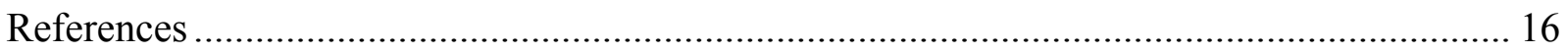

Chapter 2 Design and Simulation of Multi-Phase Buck Converter with TMU ....................... 19

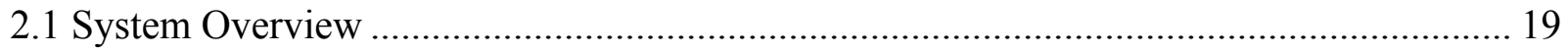

2.2 Lumped Parameter Thermal Model .................................................................... 20

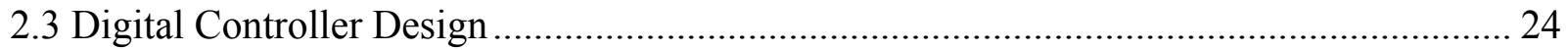

2.3.1 Voltage and Current Loop Control ................................................................ 24

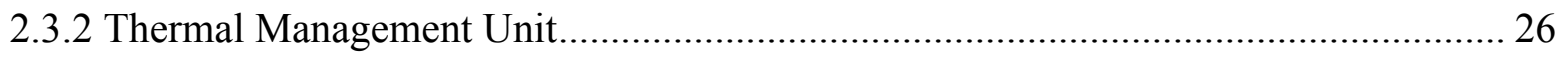

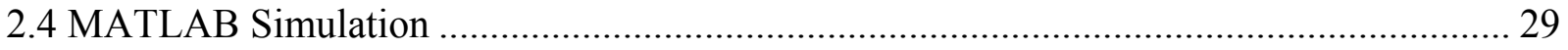

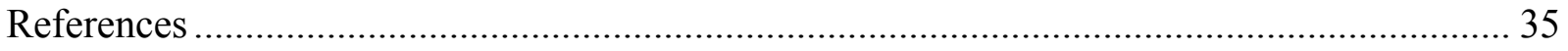

Chapter 3 Implementation and Experimental Results.................................................. 36

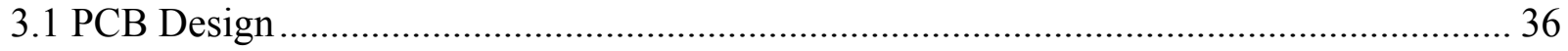

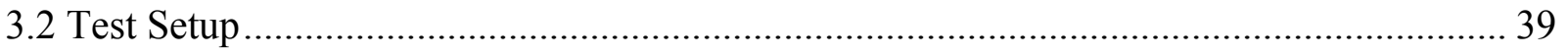

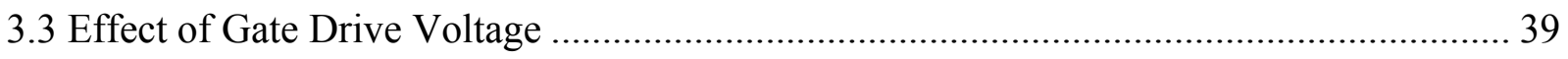


3.4 Power Efficiency Measurements....................................................................................... 40

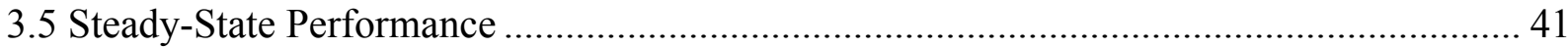

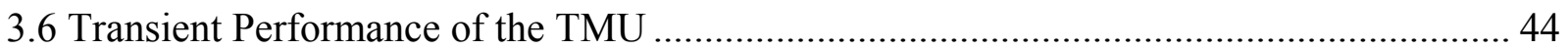

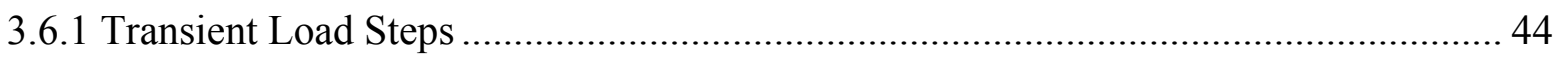

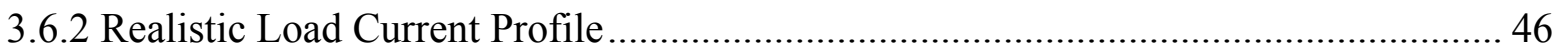

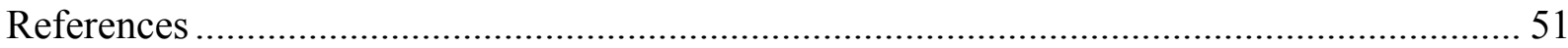

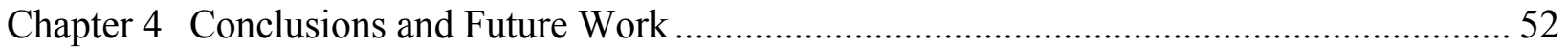

4.1 Thesis Summary …………………………………………………………………... 52

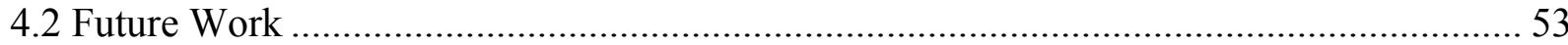

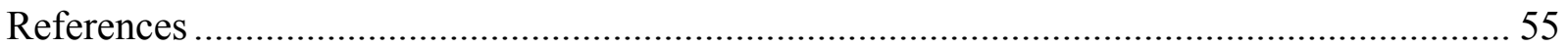

Appendix A Small-Signal AC Model of CPM Buck Converter.................................................. 56

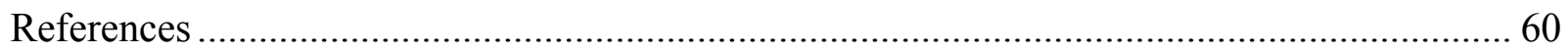

Appendix B Thermal Parameter Values Extracted for the Experimental Prototype ..................... 61 


\section{List of Tables}

Table 1.1: Voltage and Current Requirements for Intel CPUs [7]-[8] ................................... 4

Table 1.2: Prior Research Work Related to Thermal Management for Power Converters .......... 14

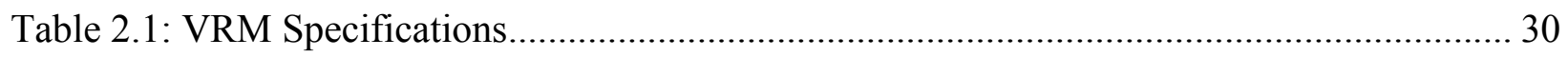

Table 3.1: Electronics Component Specifications and Comments ........................................ 37

Table 3.2: Measured Temperature and Current Data for TMU Disabled and Enabled.............. 43

Table B.1: Thermal Resistance Values Extracted for the Experimental Prototype.................... 61

Table B.2: Thermal Capacitance Values Extracted for the Experimental Prototype .................. 61 


\section{List of Figures}

Figure 1.1: Gigabyte MA78GM-S2H Motherboard with 4-phase VRMs [3].......................... 2

Figure 1.2: MSI GeForce GTX 480 Graphics Card with 16-phase VRMs [4].......................... 2

Figure 1.3: Schematic of a synchronous buck converter. .................................................. 5

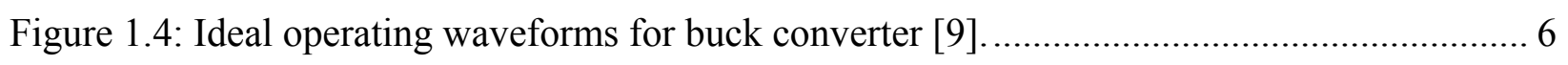

Figure 1.5: Schematic of an $n$-phase interleaved multi-phase buck converter. .......................... 7

Figure 1.6: Inductor current waveforms for a three-phase buck converter [10]..................... 8

Figure 1.7: Simplified voltage mode buck converter; VEA: Voltage Error Amplifier................ 9

Figure 1.8: Simplified average current mode buck converter; VEA: Voltage Error Amplifier;

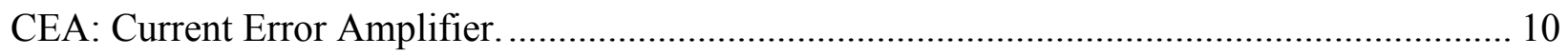

Figure 1.9: Synchronous buck converter with mixed-signal CPM...................................... 11

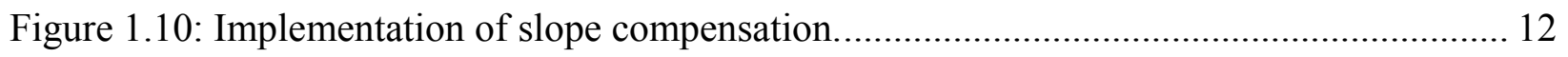

Figure 2.1: Block diagram of the four-phase interleaved CPM buck converter with TMU........ 20

Figure 2.2: Typical switching waveforms for a high-side MOSFET in a buck converter [2]..... 21

Figure 2.3: Lumped parameter thermal model for the four-phase buck converter.................... 22

Figure 2.4: Schematic of mixed-signal CPM buck converter signal phase ............................ 24

Figure 2.5: Current and HS gate signals waveforms. ...................................................... 26

Figure 2.6: Thermal model for the four-phase converter............................................... 27

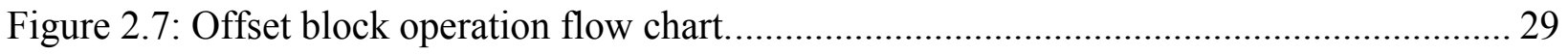

Figure 2.8: Power loss measurement of the switches and inductor for each phase................... 30

Figure 2.9: MATLAB thermal model......................................................................... 31

Figure 2.10: Normalized on-Resistance vs. Junction Temperature [10]. .................................. 32 
Figure 2.11: Simulated phase temperature with TMU disabled. 33

Figure 2.12: Simulated phase temperature with TMU enabled. 33

Figure 3.1: Fabricated mixed-signal 4 phase CPM buck converter........................................ 38

Figure 3.2: On-resistance of a power MOSFET as a function of gate to source voltage [1]....... 39

Figure 3.3: Measured temperature for one phase of the converter with the fan disabled........... 40

Figure 3.4: Measured efficiency for the four-phase converter under different operating conditions.

Figure 3.5: IR image with TMU disabled, and a total $I_{\text {out }}=40 \mathrm{~A}$. The arrow indicates the direction of airflow.

Figure 3.6: IR image with TMU enabled, with a total $I_{\text {out }}=40 \mathrm{~A}$. The arrow indicates the direction of airflow.

Figure 3.7: Temperatures measured using sensors mounted under each converter phase during a load transient with TMU disabled.

Figure 3.8: Temperatures measured using sensors mounted under each converter phase during a load transient with TMU enabled. 46

Figure 3.9: Temperatures measured during a realistic load current profile with TMU disabled.. 47

Figure 3.10: Temperature variations with TMU disabled. 47

Figure 3.11: Temperatures measured during a realistic load current profile with TMU enabled. 48

Figure 3.12: Temperature variations with TMU enabled. 48

Figure 3.13: Standard deviation of the phase temperatures with TMU disabled and enabled. .... 49

Figure A.1: Averaged small-signal ac models for buck converter operating in CCM [1]. .57 
Figure A.2: Small-signal model of the CCM CPM buck converter [1] 


\section{List of Abbreviations}

\begin{tabular}{|c|c|}
\hline ACMC & Average Current Mode Control \\
\hline ADC & Analog to Digital Converter \\
\hline CPU & Central Processing Unit \\
\hline CPM & Current Programmed Mode \\
\hline D & Duty Ratio \\
\hline DAC & Digital to Analog Converter \\
\hline DPWM & Digital Pulse-Width Modulator \\
\hline EMI & Electromagnetic Interference \\
\hline FPGA & Field-Programmable Gate Array \\
\hline HS & High-Side \\
\hline $\mathbf{L S}$ & Low-Side \\
\hline MOSFET & Metal-Oxide-Semiconductor Field-Effect-Transistor \\
\hline MTBF & Mean-Time-Before-Failure \\
\hline PCB & Printed Circuit Board \\
\hline PI & Proportional-Integral \\
\hline TMU & Thermal Management Unit \\
\hline VRM & Voltage Regulator Module \\
\hline
\end{tabular}




\section{Chapter 1 Thermal Variations in Multi-Phase Converters}

Today's high-performance microprocessors present many challenges to their power supplies. High power consumption, low bus voltages, and fast load changes are the principal characteristics which have led to the need for a switched-mode DC-DC converters local to the microprocessor.

Multi-phase interleaved converters provide fast dynamic response, small voltage and current ripple, and high power density [1]-[2]. They have been widely adopted for low output voltage, high output current, and fast load-transient applications [2], such as the voltage regulator modules (VRMs) for the CPU and graphics cards. Figure 1.1 shows the Gigabyte MA78GM-S2H motherboard with 4-phase VRMs for the CPU [3]. Figure 1.2 shows the MSI GeForce GTX 480 Graphics Card with 16-phase VRMs [4], twelve phases for the GPU, three phases for the memory and one phase for the PLL. 


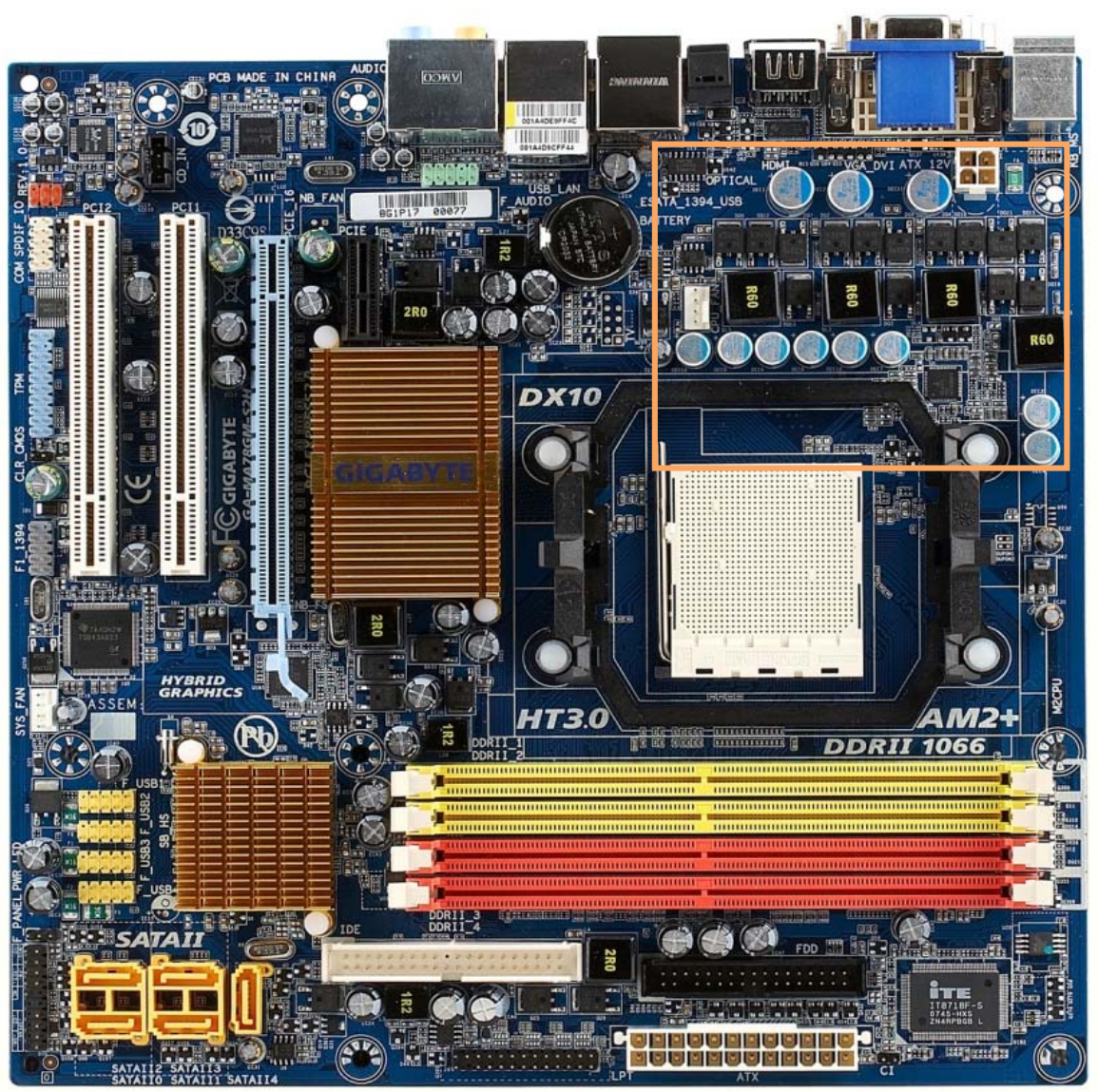

Figure 1.1: Gigabyte MA78GM-S2H Motherboard with 4-phase VRMs [3].

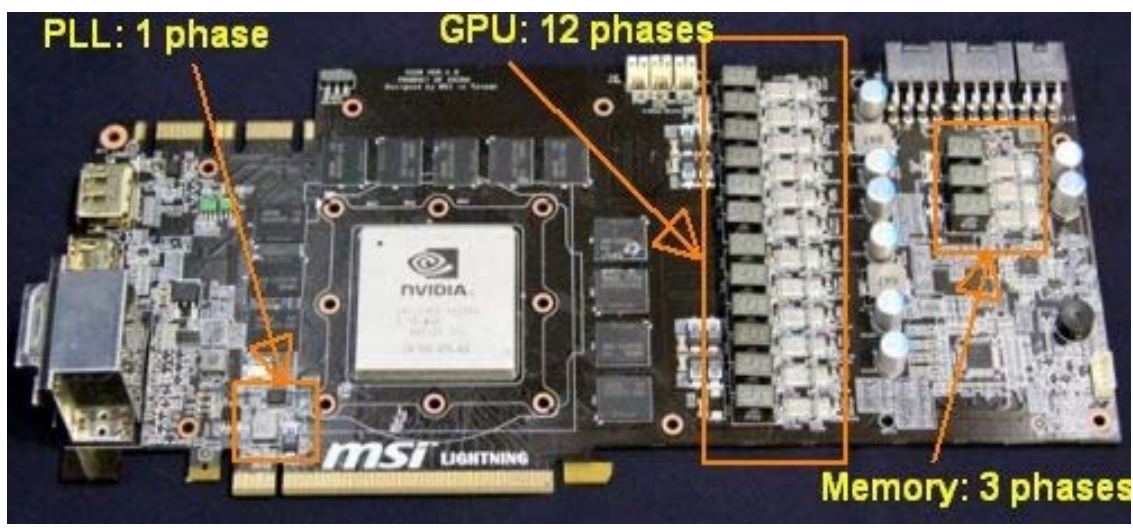

Figure 1.2: MSI GeForce GTX 480 Graphics Card with 16-phase VRMs [4]. 
In conventional designs, precise current sharing has been emphasized to reduce voltage and current ripple, while presumably minimizing thermal stress and maximizing reliability [2], [5]. However, due to variations in PCB layout, parasitic resistances, airflow, and semiconductor parameters, it is common to observe significant temperature variations between the converter phases, which lead to reliability issues and sub-optimal utilization of the power semiconductor device [1]. Temperature differences between phases may increase the peak temperature of the $\mathrm{PCB}$, reduce mean-time-before-failure (MTBF) and cause reliability issues [6]. Therefore, in this work, an active thermal management technique is proposed to regulate the current through each phase in order to achieve equal temperature across all phases.

In this chapter, different VRM design topologies are discussed. Some prior research work related to thermal management techniques for multi-phase converters are compared.

\subsection{Voltage Regulator Modules}

A voltage regulator module (VRM) is a buck converter that provides a microprocessor with the appropriate supply voltage. It usually converts $+5 \mathrm{~V}$ or $+12 \mathrm{~V}$ to a much lower voltage as required by the CPU, which is below $1.5 \mathrm{~V}$ for modern CPUs. The CPU supply voltage usually varies within a range, because of dynamic voltage scaling based on the load condition. The voltage and current requirements for the most recent Intel CPUs are as shown in Table 1.1. 
Table 1.1: Voltage and Current Requirements for Intel CPUs [7]-[8]

\begin{tabular}{|c|c|c|c|c|}
\hline Model & Technology & $\begin{array}{l}\text { Processor } \\
\text { Frequency }\end{array}$ & Voltage & Current \\
\hline $\begin{array}{c}\text { Intel }{ }^{\circledR} \text { Core }^{\mathrm{TM}} \text { i7-990X } \\
\text { Processor Extreme Edition } \\
\text { (Desktop) }\end{array}$ & $32 \mathrm{~nm}$ & $3.46 \mathrm{GHz}$ & $\begin{array}{c}0.800 \mathrm{~V}- \\
1.375 \mathrm{~V}\end{array}$ & $145 \mathrm{~A}$ \\
\hline $\begin{array}{c}\text { Intel } \AA \text { Xeon }{ }^{\circledR} \text { Processor } \\
\text { X7560 } \\
\text { (Server })\end{array}$ & $45 \mathrm{~nm}$ & $2.266 \mathrm{GHz}$ & $\begin{array}{c}1.2625 \mathrm{~V}- \\
1.4125 \mathrm{~V}\end{array}$ & $150 \mathrm{~A}$ \\
\hline
\end{tabular}

Power demands of the latest microprocessors from Intel, AMD, IBM, and other vendors continue to rise rapidly. Indeed, current desktop CPUs require over $130 \mathrm{~A}$ and more for server CPUs. Along with these stringent requirements come additional constraints such as cost, board area (power density), and thermal environment that lead to further design challenges.

Some popular VRM design topologies are introduced in the following subsections.

\subsubsection{Single Phase versus Multi-Phase Converters}

The most popular buck converter design is the synchronous buck converter topology as shown in Figure 1.3. 


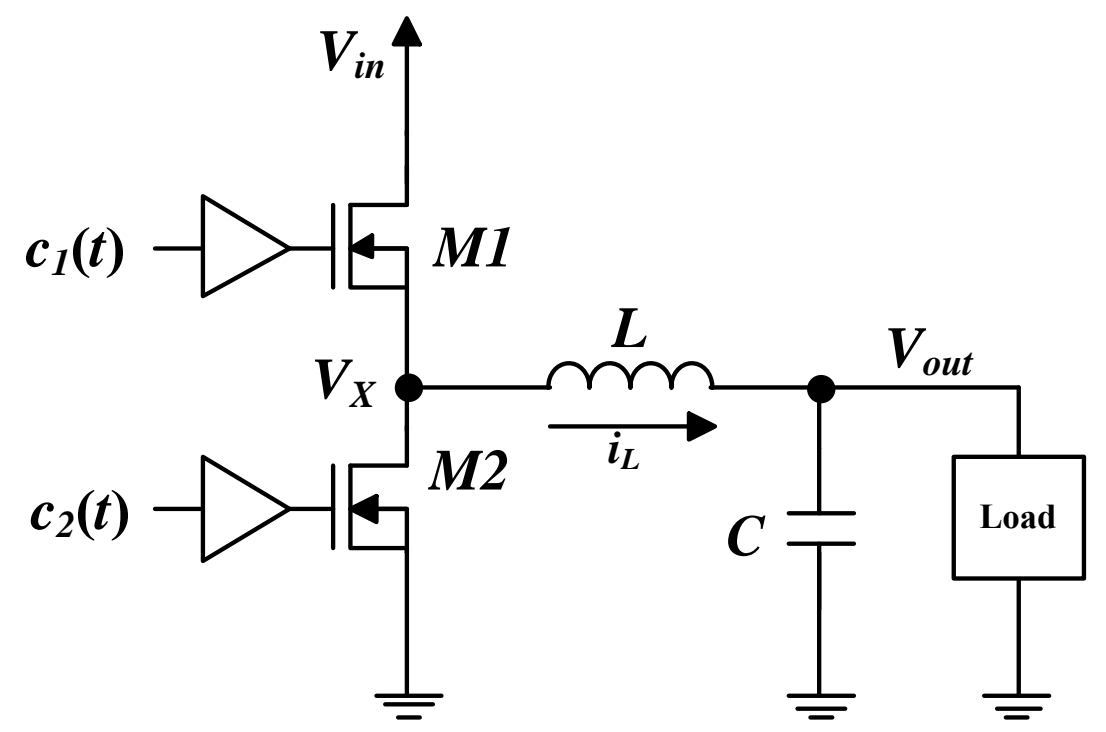

Figure 1.3: Schematic of a synchronous buck converter.

The two switches $M_{1}$ and $M_{2}$ are implemented using power MOSFETs. The synchronous topology is widely used due to its minimal voltage stress on the power devices and simple conversion ratio:

$$
M=\frac{V_{\text {out }}}{V_{\text {in }}}=D
$$

where $D$ is the duty cycle of the PWM control pulse. The high-side (HS) switch $M_{1}$ is driven by the gate driver with a duty cycle equal to $D$, while the low-side (LS) switch $M_{2}$ is driven in a complementary fashion with a duty cycle equal to $(1-D)$. The ideal operating waveforms of the synchronous converter are as shown in Figure 1.4. The switching frequency, $f_{s}$, is equal to $1 / T_{s}$, where $T_{S}$ is the switching period. 


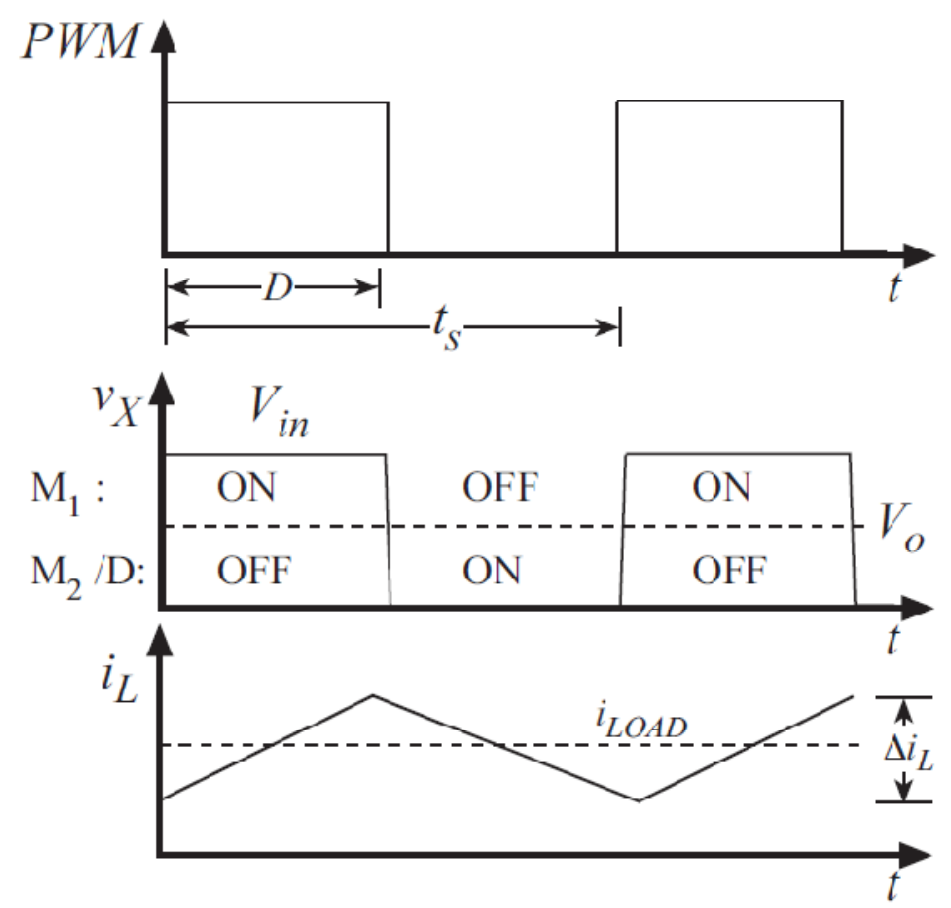

Figure 1.4: Ideal operating waveforms for buck converter [9].

The fundamental limitation of this conventional single-phase buck converter is the trade-off between efficiency and switching frequency. The output voltage ripple and dynamic response improve with increased switching frequency. The physical size and value of the filter inductor and capacitors become smaller at higher switching frequencies. However, the switching losses increase with the frequency, and the resulting efficiency is lower at higher frequencies.

By placing multiple buck converter circuits in parallel, the multi-phase interleaved buck topology offers a solution to this problem. The smaller inductance can be used in each phase to achieve the same overall voltage ripple at the same switching frequency. A converter with $n$ phases can respond to load changes $n$ times as quickly. Thus, it is especially beneficial in rapidly changing loads, such as modern microprocessors. 


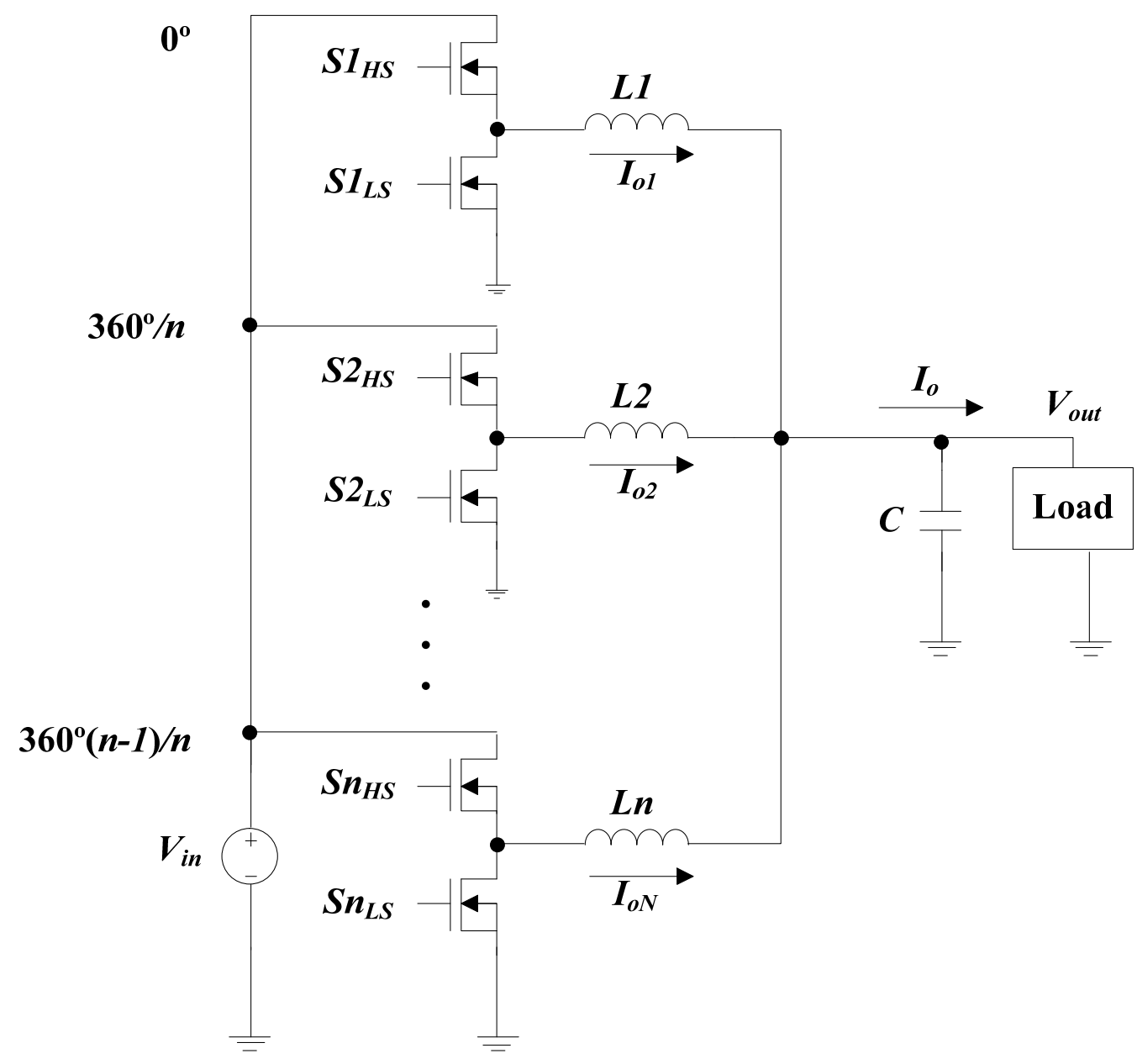

Figure 1.5: Schematic of an $n$-phase interleaved buck converter.

A multi-phase buck converter circuit is shown in Figure 1.5. Each phase is driven by gate drive signals of the same switching frequency $f_{s}$ but adjacent phases are phase shifted by $360^{\circ} / n$. For example, a three-phase converter is driven by gate signals at $0^{\circ}, 120^{\circ}$, and $240^{\circ}$ apart. The input and output of each buck cell are connected in parallel such that the fundamental ripple frequency at the input and output is $n \times f_{s}$.

There is also an $\mathrm{n}$ times decrease in switching ripple due to the increased effective frequency. The inductor current waveforms are shown in Figure 1.6 for a three-phase buck converter running at $500 \mathrm{kHz}$. Each phase is shifted by $120^{\circ}$ from its adjacent phase. The DC output current of each 
phase, i.e., $i_{L 1}, i_{L 2}, i_{L 3}$, is $1 \mathrm{~A}$ such that total $\mathrm{DC}$ output current, $i_{L}$, is $3 \mathrm{~A}$. Each phase operates at $50 \%$ duty cycle.

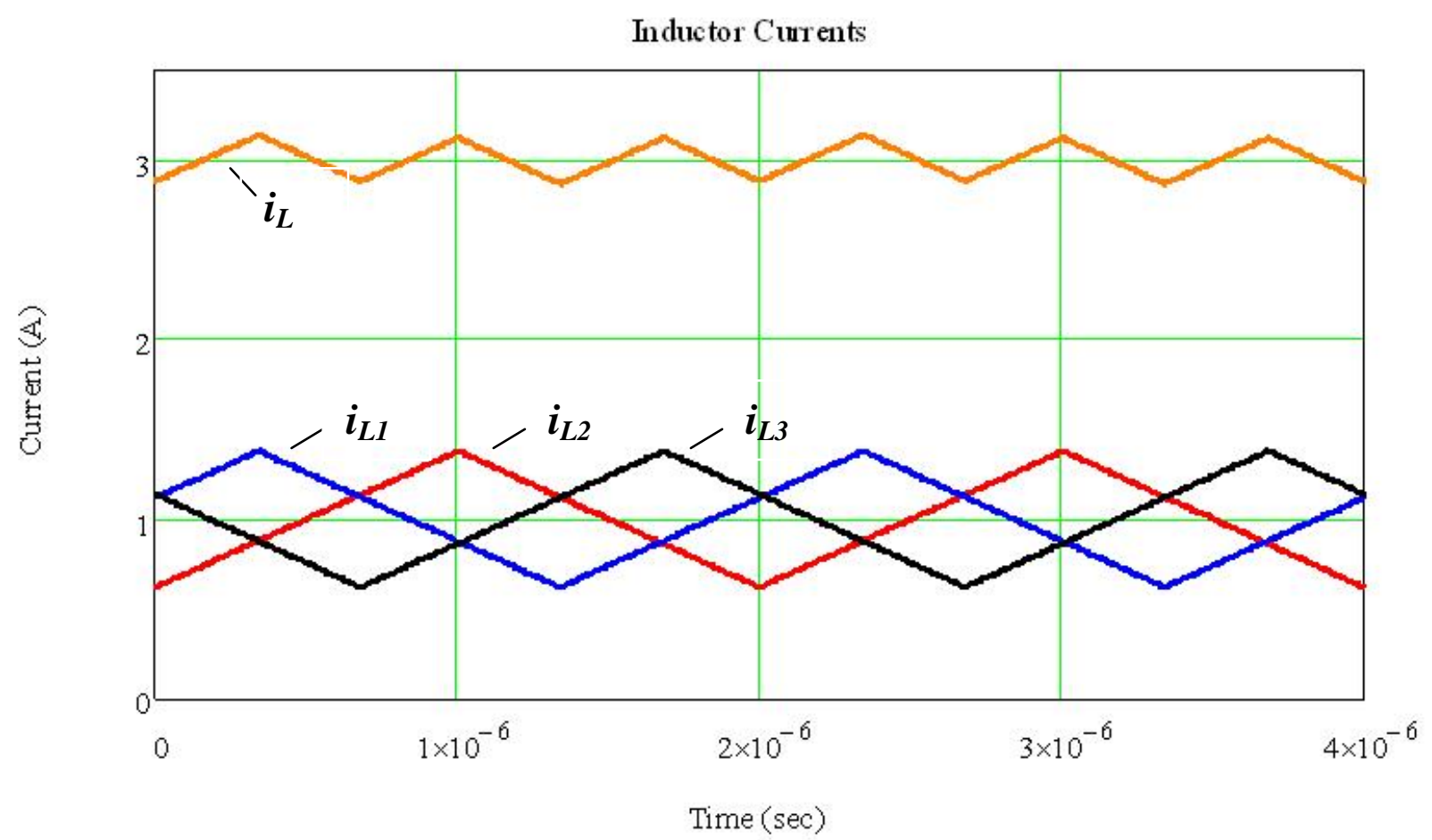

Figure 1.6: Inductor current waveforms for a three-phase buck converter [10].

Another advantage of interleaving is that the load current is shared among the $n$ phases of the multi-phase converter. This load sharing allows the heat loss to be spread out across a larger area. Hence, it helps to reduce the thermal stress in every component.

Other intrinsic advantages of this solution include reduced input and output capacitor RMS currents; reduced electromagnetic interference (EMI) filtering requirements; higher power density; the option to combine multiple filter inductors into one integrated magnetic device; better thermal and forced convection performance; and improved reliability and power stage redundancy [10]. 
In general, there are two control schemes for multi-phase converters: voltage mode and current mode control. To balance the thermal stress and properly size the power components in each phase, current sharing is critical. Voltage mode control does not have any direct control over current, which can be seen from the block diagram shown in Figure 1.7. A complicated external current sharing loop is required to achieve active current sharing, and it is usually slower than the inner voltage feedback loop.

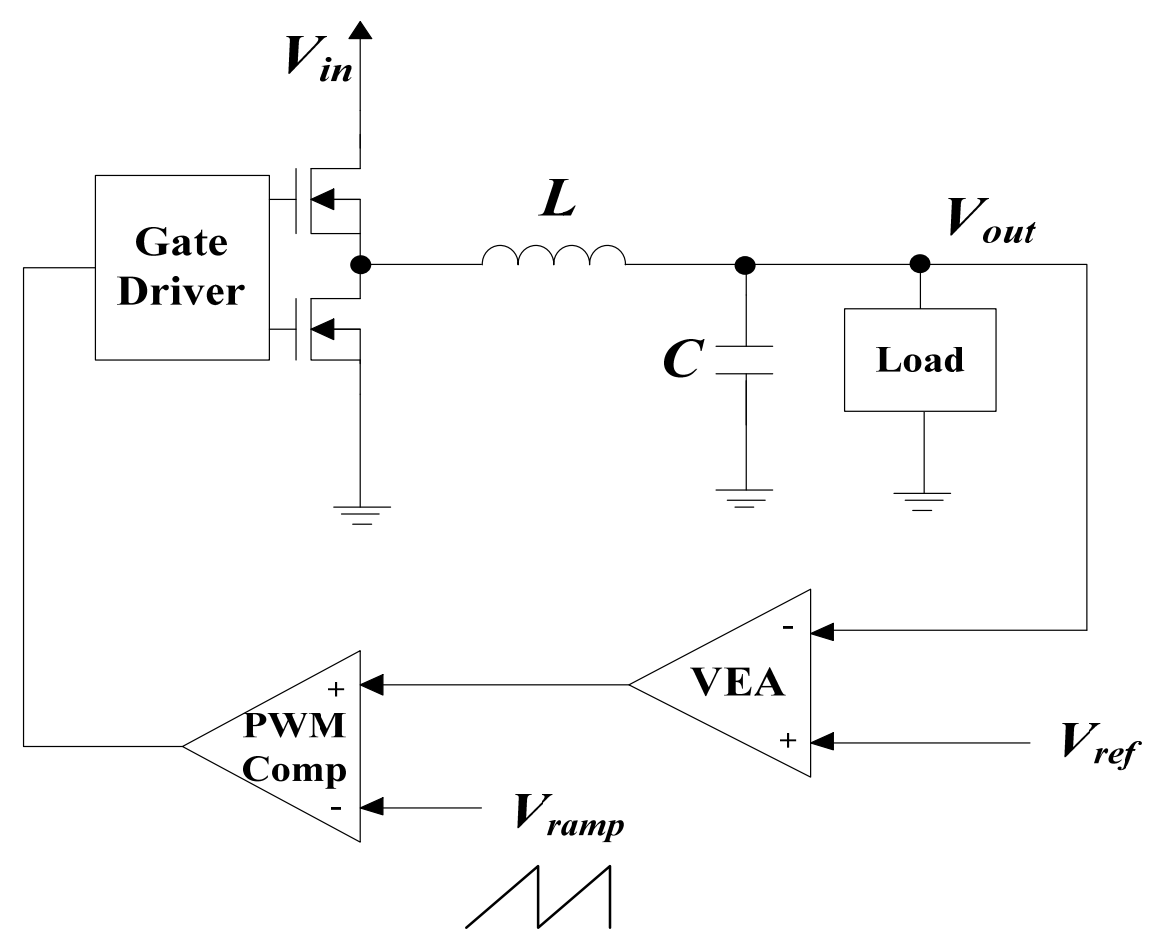

Figure 1.7: Simplified voltage mode buck converter. VEA: Voltage Error Amplifier.

In current mode control, current sharing can be easily implemented very easily with a fast current loop. There are two dominant current mode control schemes: average current mode control (ACMC) and Current Programmed Mode (CPM). ACMC especially shows good performance on precise current matching between phases. The system diagram of ACMC is shown in Figure 1.8. Although both voltage and current mode control can provide good steady-state current sharing among phases, the current mode control provides better current sharing performance and 
reliability during transients. Therefore, most VRMs are designed with current mode control. The two most common current mode control techniques, ACMC and CPM, are discussed in the next section.

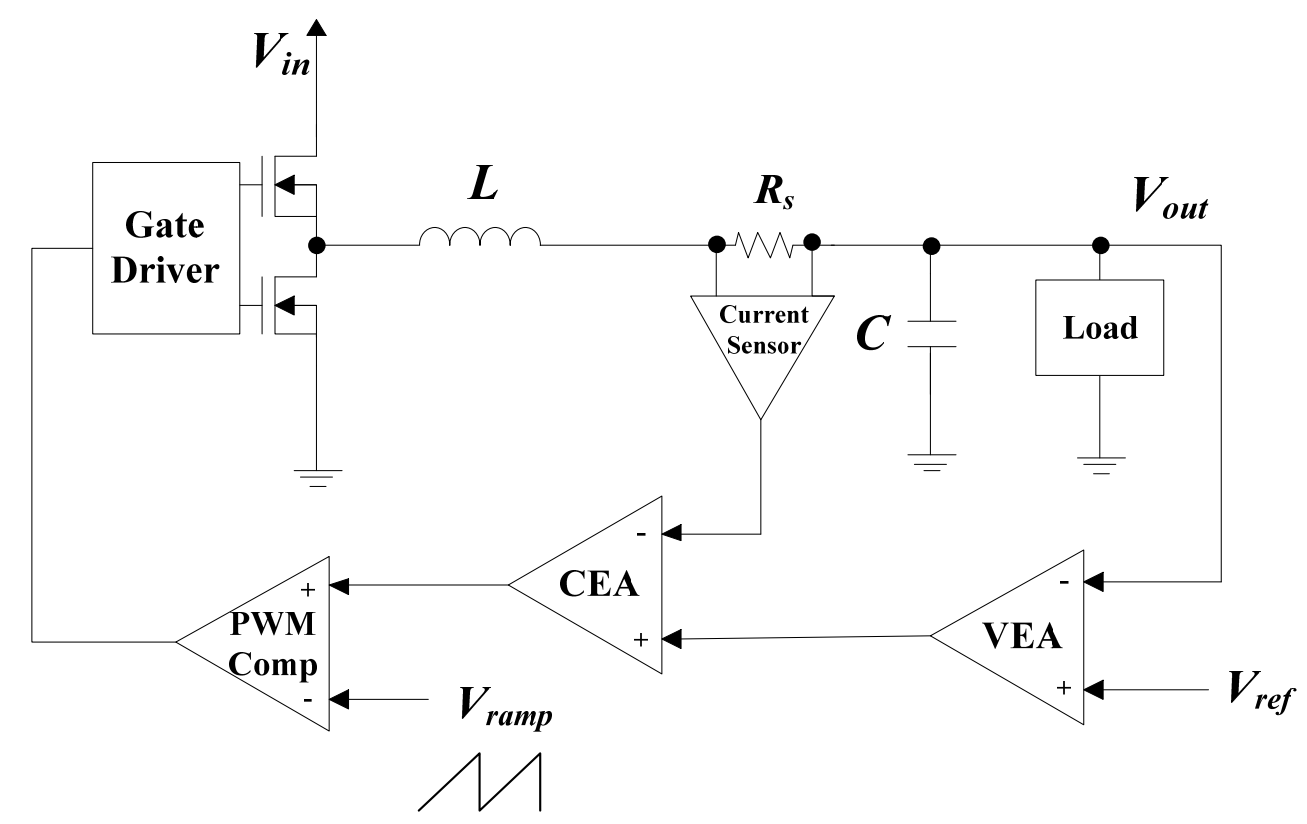

Figure 1.8: Simplified average current mode buck converter. VEA: Voltage Error Amplifier; CEA: Current Error Amplifier.

\subsubsection{Average Current Mode Control vs. Peak-Current Programmed Mode}

Current mode control is a two loop system as shown in Figure 1.9. The switched mode power supply inductor is "hidden" within the inner current control loop. This simplifies the design of the outer voltage control loop and improves power supply performance in many ways, such as better dynamics. The objective of this inner loop is to control the inductor current. In CPM, the instantaneous peak inductor current is the basis for control. 


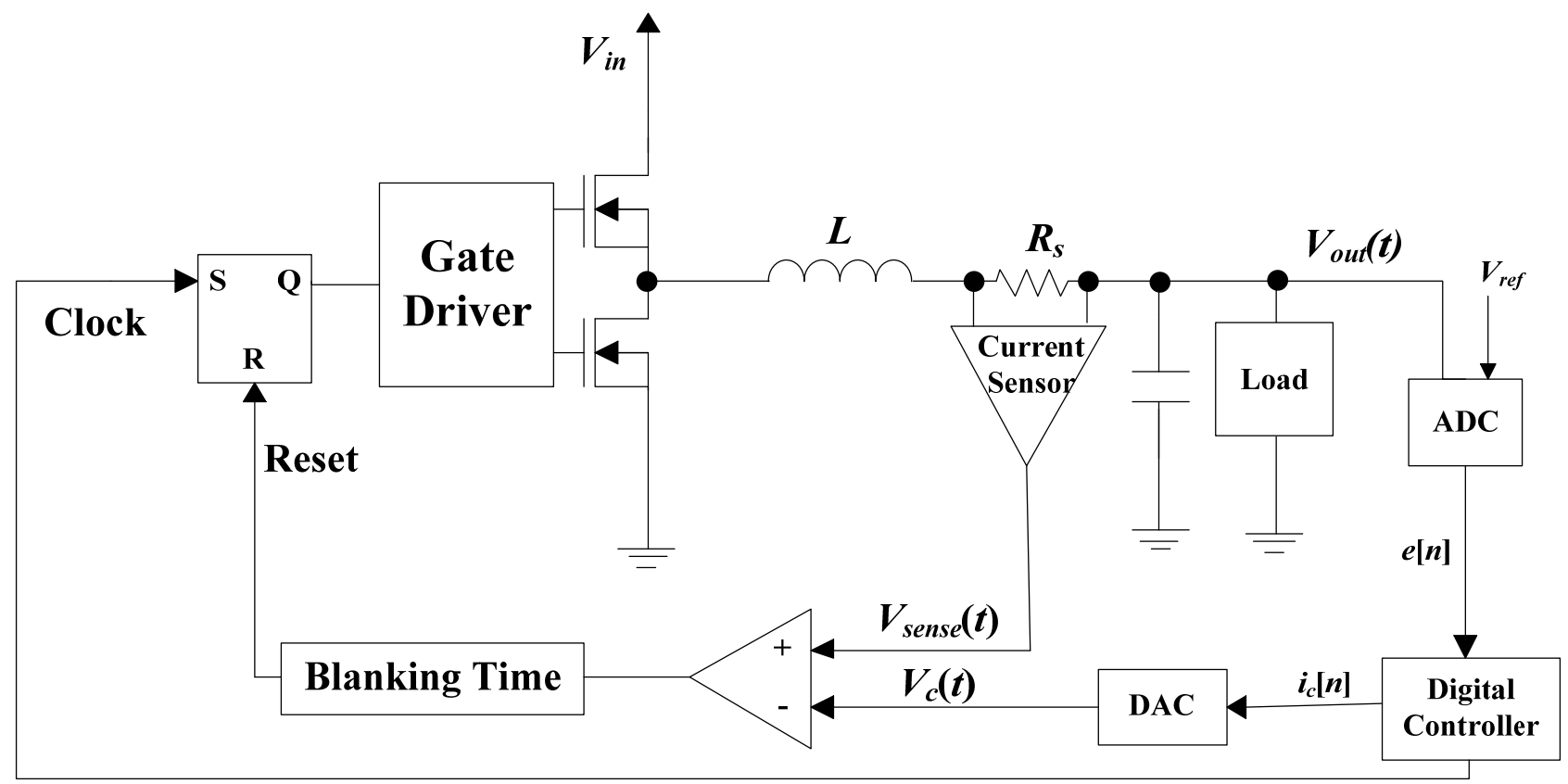

Figure 1.9: Synchronous buck converter with mixed-signal CPM.

CPM functions by comparing the rising inductor current slope to a current level set by the outer loop. The comparator turns the power switch off when the instantaneous current reaches the desired level. The current ramp is usually quite small compared to the programming level, especially when $V_{\text {in }}$ is low. As a result, this method is very susceptible to noise. A blanking time is incorporated to avoid the spurious tripping of the comparator by current spikes during switching. When the input voltage level is much higher than the output voltage level, the duty ratio $D$ is very small according to (1.1), and this gives almost no time for blanking. The duty ratio $\mathrm{D}$ needs to be at least larger than 0.05 , because the blanking time is usually designed to be approximately $5 \%$ of the switching period.

The advantages of CPM include inherent cycle-by-cycle current-limiting in the power transistors and simplified loop dynamics, which allows simple and robust compensation of the control loop [11]-[12]. The small-signal ac model of the CPM buck converter is included in Appendix A. 
In the limit where a large inductor is used, CPM approaches ACMC. In general, ACMC has better noise immunity. When the HS MOSFET is turned on, the oscillator ramp immediately dives to its lowest, far away from the corresponding current error level at the input of the PWM comparator [13].

Another disadvantage for CPM is that slope compensation is required for duty ratio exceeding $50 \%$ [11]. CPM is inherently unstable at duty ratios exceeding $50 \%$, resulting in sub-harmonic oscillation. A compensating ramp is usually applied to the comparator input to eliminate this instability, as shown in Figure 1.10. In a buck converter, the falling inductor current slope, $m_{2}$, is given by $m_{2}=V_{\text {out }} / L$. With $V_{\text {out }}$ constant, the compensating ramp slope, $m_{a}$, is fixed and easy to calculate. One common choice of $m_{a}$ is $m_{2} / 2$, which is the minimum value of $m_{a}$ that leads to stability for all duty cycles.

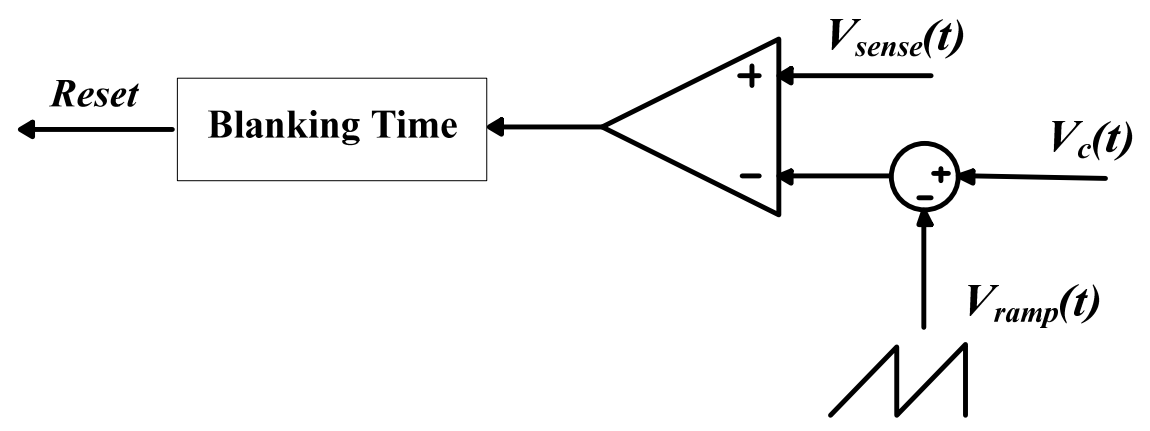

Figure 1.10: Implementation of slope compensation.

\subsection{Thermal Management for Multi-Phase Converters}

In this section, prior research work related to thermal management for power converters is briefly compared. The objective of this thesis is also discussed. 


\subsubsection{Literature Survey}

Some prior related research work is summarized in Table 1.2. In 2002, Elbanhawy et al. were granted a patent on current sharing in multi-phase converters using temperature equalization [14]. They proposed to measure the phase temperature as represented by the voltage from a temperature sensor embedded in the MOSFET gate driver placed in the immediate vicinity of the switching MOSFETs. A simple circuit compares temperatures from all phases and places the highest phase temperature on a temperature sharing bus. The local temperature within each phase is compared to this reference and the error is added to the error voltage of the PWM control loop. The combined voltage and temperature error signal is then used to adjust the phase duty cycle [15]. One major drawback of this approach is that the voltage and thermal compensation cannot be independently optimized. The voltage loop has to slow down to compensate for the thermal loop.

In 2004, Nesgaard et al. introduced a series resistor droop technique, where a thermistor was added into the feedback network. The series resistors were used to determine the output voltage level. Since one of the resistors was a thermistor, a voltage droop of each phase was created based on the phase temperature. Off-the-shelf converters were used to verify the technique experimentally [6], [16].

In 2010, Lukic et al. introduced a fully-digital thermal management scheme, where the conduction losses in each phase are calculated based on a sensor-less estimation approach. The desired load current ratio is calculated based on the estimated thermal losses [17]-[18].

In this work, we designed independent linear compensators for the thermal loops that work in conjunction with the existing voltage control loop. The thermal linear compensators regulate the 
Chapter 1. Thermal Variations in Multi-Phase Converters

current through each phase to achieve equal temperature across all phases. Explicit temperature sensors were used in each phase to achieve accurate temperature feedback.

Table 1.2: Prior Research Work Related to Thermal Management for Power Converters

\begin{tabular}{|l|c|c|c|l|}
\hline \multicolumn{1}{|c|}{ Related Work } & $\begin{array}{c}\text { Temperature } \\
\text { Measurement }\end{array}$ & Control & Phases & Technique \\
\hline $\begin{array}{l}\text { A. Elbanhawy } \\
\text { U.S. Patent, 2002 } \\
\text { [14] } \\
\text { PESC 2005 [15] }\end{array}$ & Sensor & $\begin{array}{c}\text { Voltage mode } \\
\text { PWM (analog) }\end{array}$ & 2 & $\begin{array}{l}\text { Adding temperature error } \\
\text { signal to PWM control loop } \\
\text { error voltage }\end{array}$ \\
\hline $\begin{array}{l}\text { C. Nesgaard } \\
\text { PESC 2004 [16] }\end{array}$ & Sensor \\
EPE 2005 [6] & $\begin{array}{c}\text { off-the-shelf } \\
\text { converter (analog) }\end{array}$ & $2 ; 3$ & Thermal droop load sharing \\
\hline $\begin{array}{l}\text { Z. Lukic } \\
\text { 2010 [17]-[18] }\end{array}$ & $\begin{array}{c}\text { Sensor-less } \\
\text { ACMC (digital) }\end{array}$ & 2 & $\begin{array}{l}\text { Calculating desired current } \\
\text { ratio based on estimated } \\
\text { phase resistances }\end{array}$ \\
\hline This work [19] & Sensor & CPM (digital) & 4 & $\begin{array}{l}\text { Independent linear thermal } \\
\text { loop controllers }\end{array}$ \\
\hline
\end{tabular}

\subsubsection{Thesis Objectives}

In this thesis, an active thermal management control scheme for multi-phase current mode buck converters is proposed and investigated. This control scheme incorporates a thermal management unit (TMU) into the existing digital control loop to regulate the current through each phase, in 
order to achieve temperature equalization across all phases. The goal of this work includes the following tasks:

- Evaluate the performance of this thermal management technique through simulations and experiments. The performance evaluation includes steady-state performance, transient performance, efficiency, etc.

- Look beyond the steady-state temperature equalization by investigating the dynamic transient response of the thermal system running in closed loop.

- Verify the effectiveness of the TMU under extreme cases. Non-uniform airflow distribution is used to create significant temperature variations between phases.

\subsection{Thesis Organization}

Implementation details of the TMU and system-level simulation results are provided in Chapter 2. The experimental results are reported in Chapter 3. Finally, the summary of the thesis and potential ideas for future work are provided in Chapter 4. 
Chapter 1. Thermal Variations in Multi-Phase Converters

\section{References}

[1] Z. Lukic, S. M. Ahsanuzzaman, and A. Prodic, "Self-Tuning Sensorless Digital CurrentMode Controller with Accurate Current Sharing for Multi-Phase DC-DC Converters," in Proc. APEC 2009, pp. 264 - 268, Washington, DC, Feb. 15-19, 2009.

[2] P. L. Wong, P. Xu, B. Yang, and F. C. Lee, "Performance Improvements of Interleaving VRMs with Coupling Inductors," IEEE Trans. Power Electronics, vol. 16, no. 4, pp. 499507, July 2001.

[3] Gigabyte MA78GM-S2H Motherboard. Image available: http://ixbtlabs.com/articles3/mainboard/gigabyte-ma78gm-s2h-780g-p1.html

[4] MSI GeForce GTX 480 Graphics Card. Image available: http://www.geeks3d.com/20100917/msi-gtx-480-lightning-16-phase-vrm-and-3-powerconnectors/

[5] Y. Panov and M. M. Jovanovic, "Stability and Dynamic Performance of Current-Sharing Control for Paralleled Voltage Regulator Modules," IEEE Trans. Power Electronics, vol. 17, no. 2, pp. 172-179, March 2002.

[6] C. Nesgaard, and M.A.E. Andersen, "Experimental Verification of the Thermal Droop Load Sharing Technique," in Proc. EPE 2005, pp. P1-P10, Dresden, Germany, Sep. 11-14, 2005.

[7] Intel Corporation, "Intel ${ }^{\circledR}$ Core $^{\mathrm{TM}}$ i7-900 Desktop Processor Extreme Edition Series and Intel® CoreTM i7-900 Desktop Processor Series on 32-nm Process”, July 2010.

[8] Intel Corporation, “Dual-Core Intel ${ }^{\circledR} \operatorname{Xeon}^{\circledR}$ Processor 7000 Series”, Revision 2.1, September 2006. 
Chapter 1. Thermal Variations in Multi-Phase Converters

[9] O. Trescases, “A High-Frequency, Soft-Switching DC-DC Converter for Dynamic Voltage Scaling in VLSI Loads", Master Thesis, 2004.

[10] A. Elbanhawy, "Current Sharing in a Multi-phase Power Supply by Phase Temperature Control,” U.S. Patent 6,449,174 B1, Sep. 10, 2002.

[11] R. Erickson and D. Maksimovic, Fundamentals of Power Electronics. Norwell, MA: Kluwer, 2001.

[12] O. Trescases, A. Prodic, and W. T. Ng, "Digitally Controlled Current-Mode DC-DC Converter IC," IEEE Transactions on Circuits and Systems, vol. 58, no. 1, pp. 219-231, January 2011.

[13] L. Dixon, “Average Current Mode Control of Switching Power Supplies,” Unitrode Application Note, pp. 356-369, 1990.

[14] T. Hegarty, "Benefits of Multiphasing buck converters - Part 1”, EE Times, November 2007.

[15] A. Elbanhawy, "Current Sharing in Multiphase Converters Using Temperature Equalization,” in Proc. PESC 2005, pp. 1464 - 1468, Recife, Jun. 16-19, 2005.

[16] C. Nesgaard and M.A.E. Andersen, "Optimized Load Sharing Control by means of Thermal Reliability Management," in Proc. PESC 2004, pp. 4901-4906, Aachen, Germany, Jun. 20$25,2004$.

[17] Z. Lukic, "Design and Practical Implementation of Advanced Reconfigurable Digital Controllers for Low-Power Multi-Phase DC-DC Converters," Ph.D. thesis, University of Toronto, Canada, 2010. 
Chapter 1. Thermal Variations in Multi-Phase Converters

[18] Z. Lukic, S M Ahsanuzzaman, Z. Zhao, and A. Prodic, "Sensorless Self-Tuning Digital CPM Controller with Multiple Parameter Estimation and Thermal Stress Equalization", Submitted to IEEE Trans. Power Electron.

[19] K. Cao, W. T. Ng, and O. Trescases, "Thermal Management for Multi-Phase Current Mode Buck Converters", in Proc. APEC 2011, March 2011. 


\section{Chapter 2 Design and Simulation of Multi-Phase Buck Converter with TMU}

In this chapter, the verification of the proposed thermal management unit (TMU) with MATLAB Simulink is described. The performance of a mixed-signal four-phase interleaved buck converter with TMU was simulated and is discussed. A lumped parameter thermal model for the converter was developed to monitor the dynamic temperature distribution. The dynamic temperature feedback was incorporated into a MATLAB simulation model. In the following sections, the overall system is introduced, the lumped parameter thermal model is discussed, and the digital controller design including the TMU is explained. Finally, MATLAB simulation results are presented.

\subsection{System Overview}

A $12 \mathrm{~V}$ to $1 \mathrm{~V}, 50 \mathrm{~A}, 250 \mathrm{kHz}$, four-phase mixed-signal CPM buck converter was designed to verify the TMU concept. Four-phase converter was chosen for the purpose of TMU demonstration. The system block diagram of the converter with the digital controller is as shown in Figure 2.1. In this work, precise current sharing is not required, since the TMU generates a current offset for each phase to achieve equal temperature across all phases. Hence, CPM was used instead of ACMC to allow for faster transient response and inherent cycle-by-cycle current limiting. A peak current control loop is implemented in each phase. Since the duty cycle is well below $50 \%$, slope compensation is not needed, as discussed in Chapter 1.

The proposed control system consists of a centralized digital proportional-integral (PI) compensator that regulates the output voltage, $v_{\text {out }}(t)$ and a TMU that regulates the phase 
temperatures, $T_{1-4}$. Temperature sensors are located near the power stage in each phase and the sampled temperature data is transmitted to the TMU using a shared serial bus using SMBus protocol. The chosen TMP275 temperature sensors provide $0.5{ }^{\circ} \mathrm{C}$ accuracy and $\pm 0.0625{ }^{\circ} \mathrm{C}$ resolution [1]. The gate-driver IC is powered by a separate voltage supply in order to emulate $R_{O N}$ variations in the experimental setup.

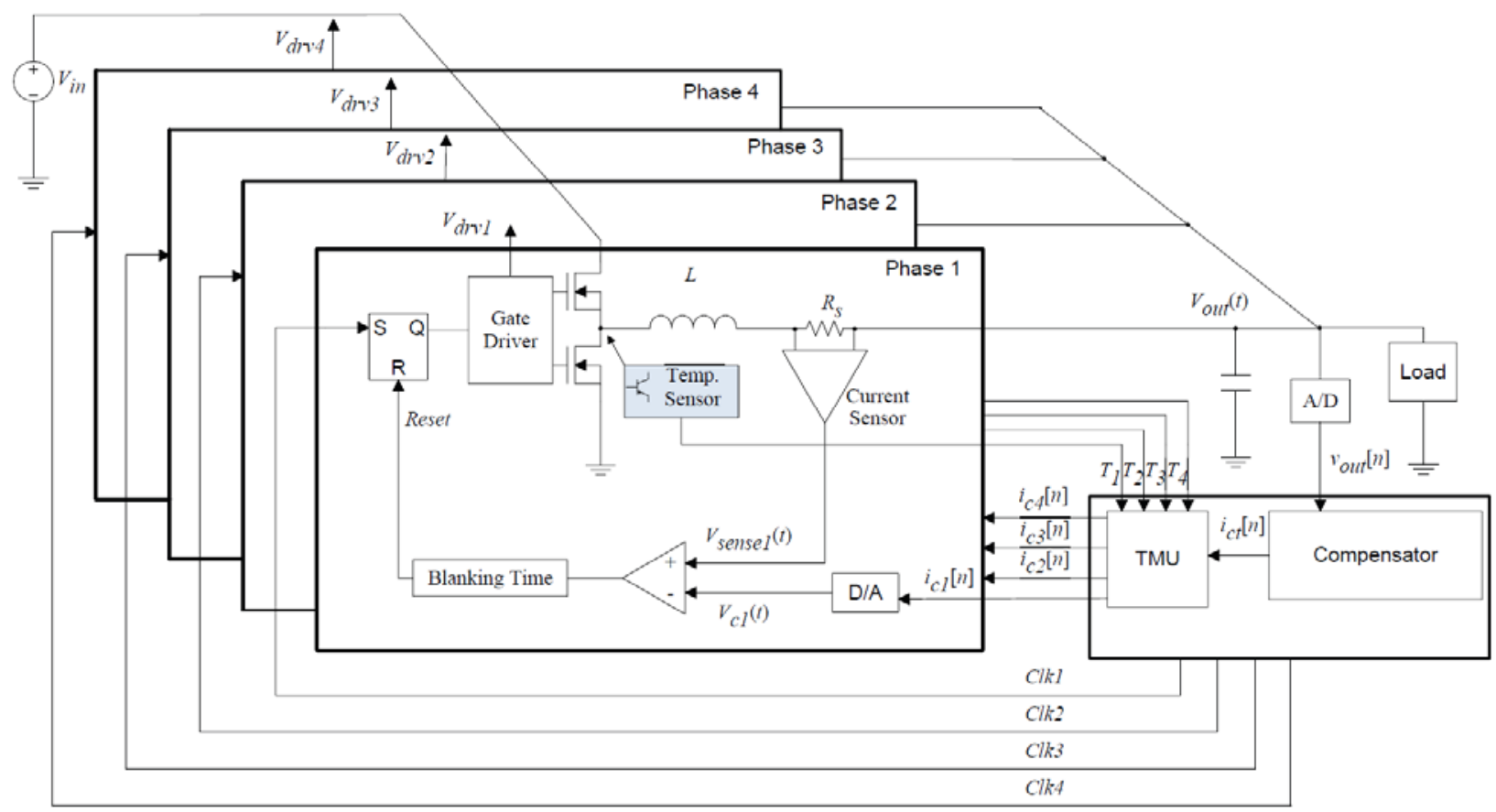

Figure 2.1: Block diagram of the four-phase interleaved CPM buck converter with TMU.

\subsection{Lumped Parameter Thermal Model}

In each phase, the HS MOSFET, the LS MOSFET and the inductor are considered to be the dominant power losses. The other losses caused by the inductor DC resistance (DCR) and capacitor equivalent series resistance (ESR) are relatively small. Typical switching waveforms for the HS MOSFET of the synchronous buck converter in Figure 1.3 are as shown in Figure 2.2. 


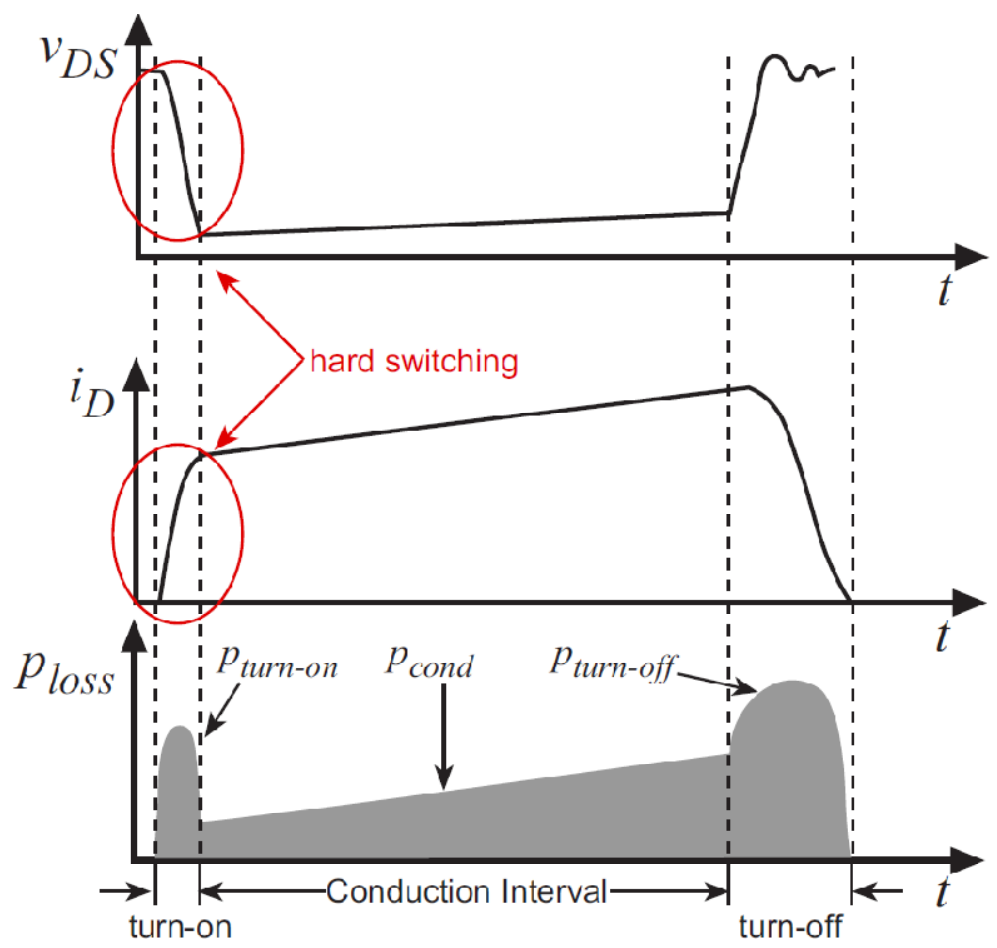

Figure 2.2: Typical switching waveforms for a high-side MOSFET in a buck converter [2].

For the HS and LS MOSFETs, the main power losses are switching losses (3.1) and conduction losses $(3.2,3.3)$. For the inductor, the power losses are mainly conduction losses (3.4). The switching losses of the power MOSFETs, $P_{s w}$ is given by [3]:

$$
P_{s w}=P_{\text {turn-on }}+P_{\text {turn-off }} \approx 2 C_{x} V_{\text {in }}^{2} f_{S}
$$

where $P_{\text {turn-on }}$ is the power loss during the turn-on interval, $P_{\text {turn-off }}$ is the power loss during the turn-off interval and $C_{x}$ is the capacitance at the switching node $V_{x}$. The conduction losses, $P_{\text {cond }}$ in the power MOSFETs and the inductor are given by:

$$
\begin{gathered}
P_{\text {Cond_HS }}=I_{L O A D}{ }^{2} \cdot D \cdot R_{d s_{-} H S} \\
P_{\text {Cond_LS }}=I_{L O A D}{ }^{2} \cdot(1-D) \cdot R_{d S_{-} L S}
\end{gathered}
$$


Chapter 2. Design and Simulation of Multi-Phase Buck Converter with TMU

$$
P_{\text {cond_ind }}=I_{L O A D}{ }^{2} \cdot R_{D C R}
$$

where $R_{d S_{-} H S}, R_{d S_{-} L S}$ are the on-resistance of the HS and LS MOSFETs respectively, and $R_{D C R}$ is the DC resistance of the inductor.

A lumped element thermal model for the VRM is as shown in Figure 2.3. This thermal model is a simplified version to be used as a starting point for the TMU design. The three dominant power losses in each phase, the high-side transistor loss, the low-side transistor loss and the inductor loss are denoted as $P_{H S}, P_{L S}$ and $P_{I n d}$, respectively.

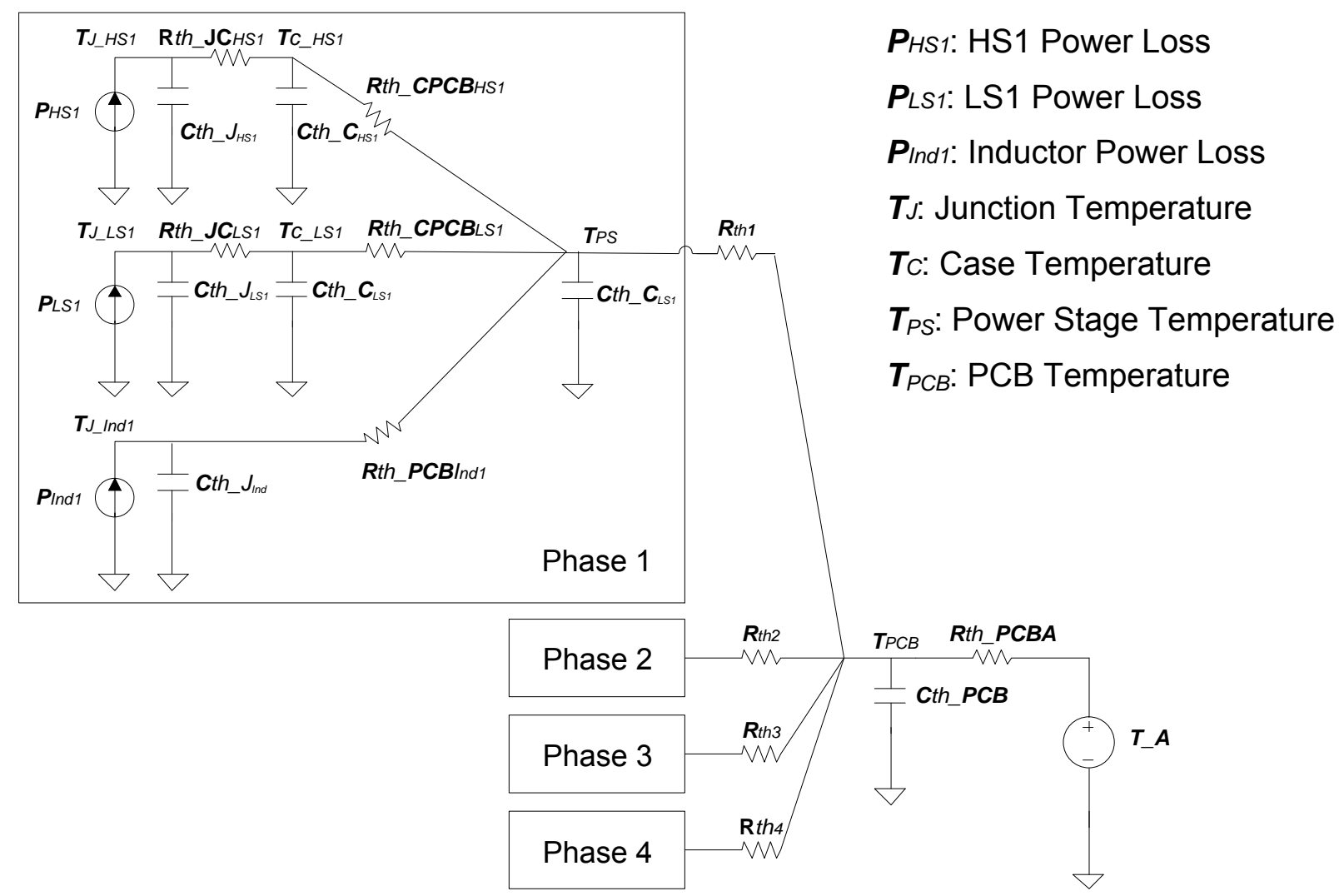

Figure 2.3: Lumped parameter thermal model for the four-phase buck converter. 
The thermal coupling between the different converter phases is modeled by the thermal resistances, $R_{t h 1-3}$. Thermal capacitances are included in the model to account for the thermal time constants in the real system.

Several thermal parameters were taken from the datasheets for various components, such as the junction to case thermal resistances of the power MOSFETs $R_{t h \_} J C_{H S}$ and $R_{t h \_} J C_{L S}$. The remaining parameters were extracted using semi-empirical methods during the experimental phase. The power losses of the HS MOSFET $\left(P_{H S}\right)$, LS MOSFET $\left(P_{L S}\right)$ and the inductor $\left(P_{\text {Ind }}\right)$ can be calculated using (3.1) to (3.4). A FLIR T300 infrared (IR) camera was used to measure the temperature at different points of the system, such as the MOSFET case temperature, the inductor temperature and the PCB temperature. The extracted thermal parameter values are listed in Appendix A.

Commercial software packages are available for fine-grained PCB thermal analysis using finite element methods [4]. These CAD tools can provide both steady-state and transient thermal profiles. They are useful for component placement and PCB design. However they cannot be easily integrated into closed-loop system-level simulators for switched-mode power supplies, as required in this work.

The lumped parameter model in Figure 2.3 was used as a starting point for the design and simulation of the TMU. A detailed description is given in the following section. 


\subsection{Digital Controller Design}

The proposed control system as shown in Figure 2.1 consists of a centralized digital PI compensator to regulate $v_{\text {out }}(t)$ and a TMU to regulate the phase temperatures, $T_{1-4}$. In this section, the voltage and current control loop designs are introduced. The TMU design, which is the focus of this thesis, is discussed in detail. The small-signal ac model is included in Appendix B.

\subsubsection{Voltage and Current Loop Control}

Mixed-signal CPM [5], [6] is a hybrid control scheme, where voltage-loop compensation is carried out in the digital domain, while the current-regulation loop is a traditional analog implementation. The schematic of a single phase converter is as shown in Figure 2.4. Using this architecture, a DAC is required at the interface between these two loops, in order to generate an analog current command.

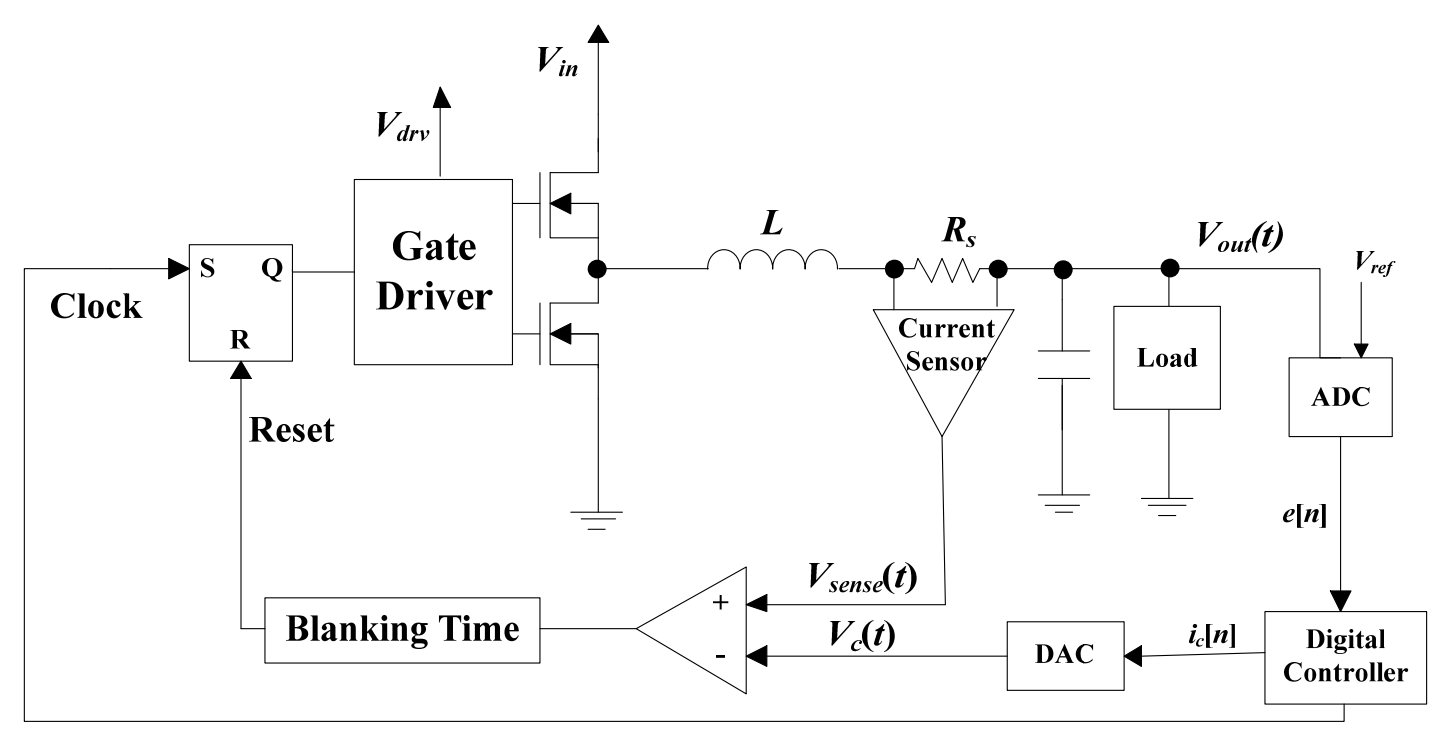

Figure 2.4: Schematic of mixed-signal CPM buck converter signal phase. 
The output voltage is compared with the voltage reference, and the error signal is fed into the digital proportional-integral (PI) compensator. The PI compensator outputs a digital current command, and DAC converts it to an analog current command.

A sense resistor $R_{S}$ is added after the inductor to sense the output current. In order to reduce power dissipation, a small value resistor should be used. In this work, $R_{S}$ was chosen to be $5 \mathrm{~m} \Omega$. This causes $0.5 \mathrm{~W}$ conduction losses from the inductor in each phase when the output current is $40 \mathrm{~A}$, which contributes to $1.25 \%$ of the efficiency loss. A high-bandwidth sense amplifier is connected across $R_{S}$ to produce the voltage level of the output current.

A comparator is used to compare the current sensor output, $V_{\text {sense }}$ and the DAC output. When the inductor current reaches the level of the DAC output, $v_{c}(t)$, the output of the comparator will go high and resets the SR latch. The HS MOSFET is turned on at the beginning of every switching cycle. By resetting the SR latch, the HS MOSET is turned off and the LS MOSFET is turned on for the rest of the switching cycle. The waveforms of the current and HS gate signals are as shown in Figure 2.5. The duty cycle is determined by the peak of the current through the inductor. A blanking time is incorporated to avoid the spurious tripping of the comparator by current spikes during switching. 


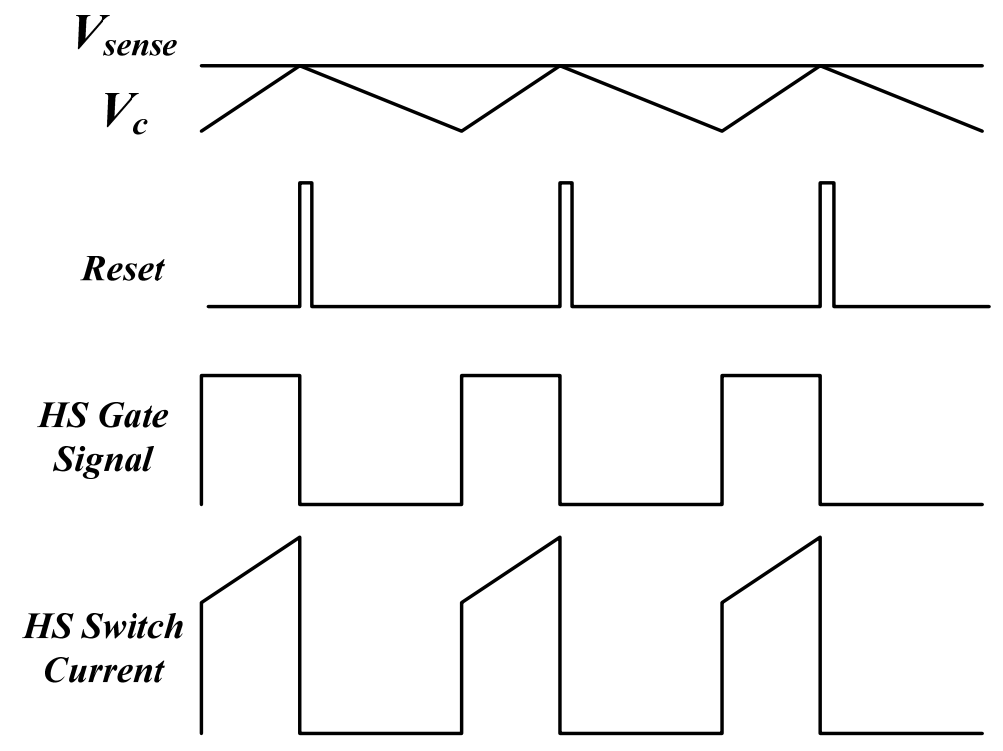

Figure 2.5: Current and HS gate signals waveforms.

Mixed-signal CPM benefits from the simplicity of the analog current loop and the flexibility of the digitally compensated voltage loop. With this approach, a reconfigurable digital compensator can be used without the need for oversampling the inductor current. The high-frequency digital pulse-width modulator (DPWM) required for fully digital CPM schemes [7]-[10] is also eliminated, resulting in a practical, low-cost implementation.

\subsubsection{Thermal Management Unit}

A thermal management unit (TMU) is designed to regulate the current through each phase in order to achieve equal temperatures across all phases. The architecture of the TMU is as shown in Figure 2.6. 


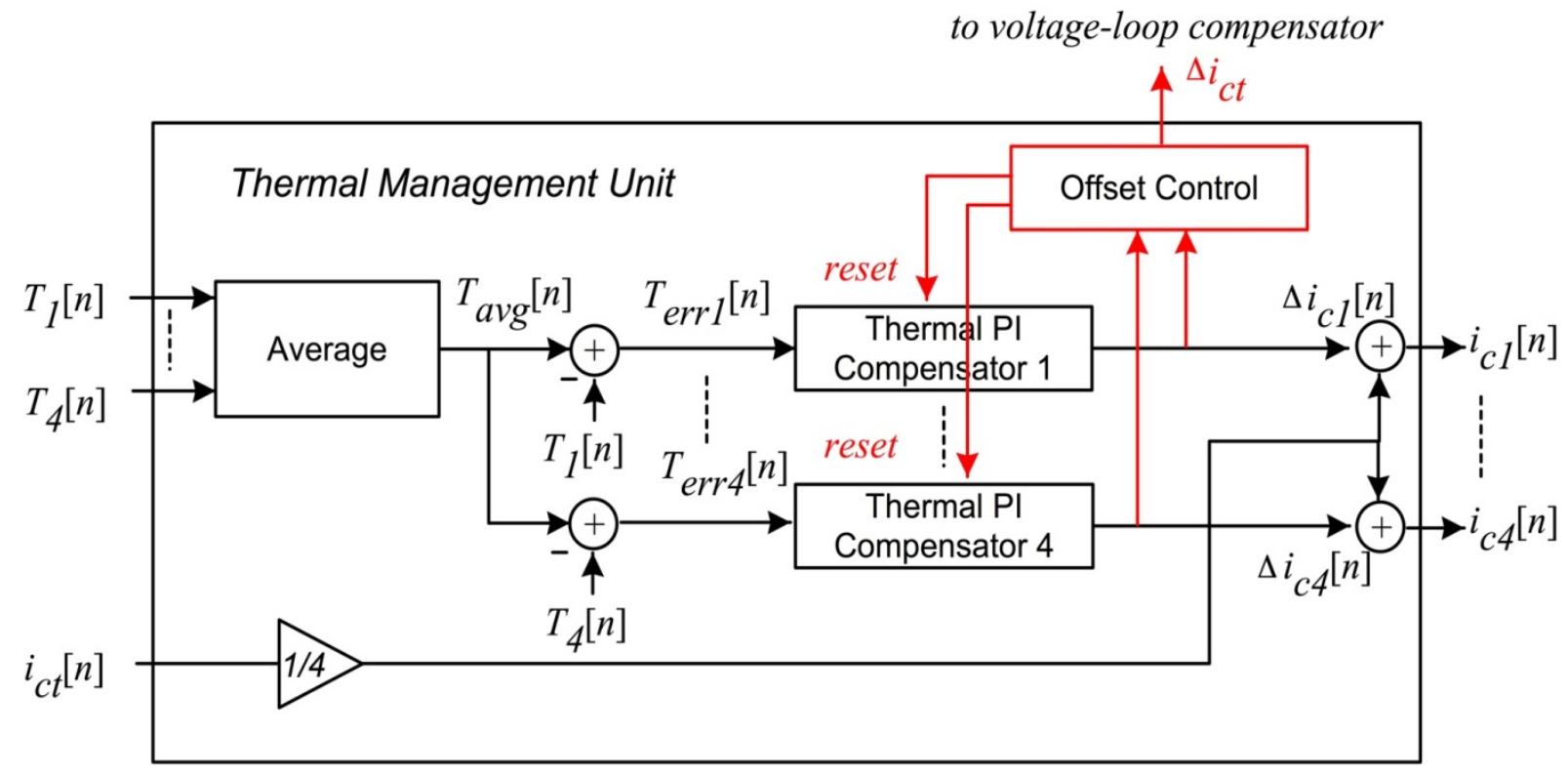

Figure 2.6: Thermal model for the four-phase converter.

After sensing the temperature from each phase, the average temperature, $T_{a v g}$ is computed. This average temperature is then used as a reference for the four independent linear PI compensators. Each PI compensator outputs a temperature offset $\Delta i_{c l-4}$. The final current commands for the CPM current loops are generated by adding the $\Delta i_{c l-4}$ to the central current command $i_{c t}$ that is generated by the voltage loop compensator.

The dynamics of the thermal loops are several orders of magnitude slower than the voltage loop due to the long thermal time constants. This effectively decouples the voltage and temperature regulation loops and simplifies the controller design. During normal operation, the voltage compensator can easily maintain regulation while the thermal PI compensators slowly adjust the current distribution. In fact, the frequency of the zero $\omega_{z t}$ in the thermal PI must be so low such that implementing the compensator with analog integrated circuits would require prohibitively large passive components. Digital implementation of the PI is much more convenient since $\omega_{z t}$ can be shifted to arbitrarily low frequencies, given a sufficient register size. The thermal PI 
compensators effectively store the current offsets needed to maintain a uniform temperature distribution. Ideally the sum of the offset currents, $\Delta i_{T}$ is zero:

$$
\sum_{i=1}^{4} \Delta i_{c i}=\Delta i_{T}=0
$$

This condition guarantees that the current-command from the voltage compensator $i_{c t}$ represents the average of the peak currents at all times. Unfortunately, this condition is not inherently guaranteed by the control scheme. In fact, $\Delta i_{T}$ may gradually grow in time as the converter runs in closed-loop with multiple load steps. This is clearly problematic since the registers in the PI compensators can overflow as $i_{c t}$ and $\Delta i_{c l-4}$ grow in opposite directions. This problem is solved by introducing an offset control block that actively monitors $\Delta i_{T}$. Upper limit threshold $\Delta i_{\text {th_max }}$ and lower limit threshold $\Delta i_{\text {th_min }}$ based on the size of the register. When $\Delta i_{T}>\Delta i_{t h}$ max , the offset control block subtracts $\Delta i_{T} / 4$ from the thermal PIs and adds $\Delta i_{T}$ to the voltage compensator's internal register. Similarly, when $\Delta i_{T}<\Delta i_{t h \_ \text {min }}$, the offset control block adds $\Delta i_{T} / 4$ to the thermal PIs and subtracts $\Delta i_{T}$ from the voltage compensator's internal register. This operation effectively resets the offset, without disturbing the actual current commands $i_{c l-4}$. A flow chart as shown in Figure 2.7 summarizes the principle of operation. This offset cancellation process happens automatically during normal operation. 


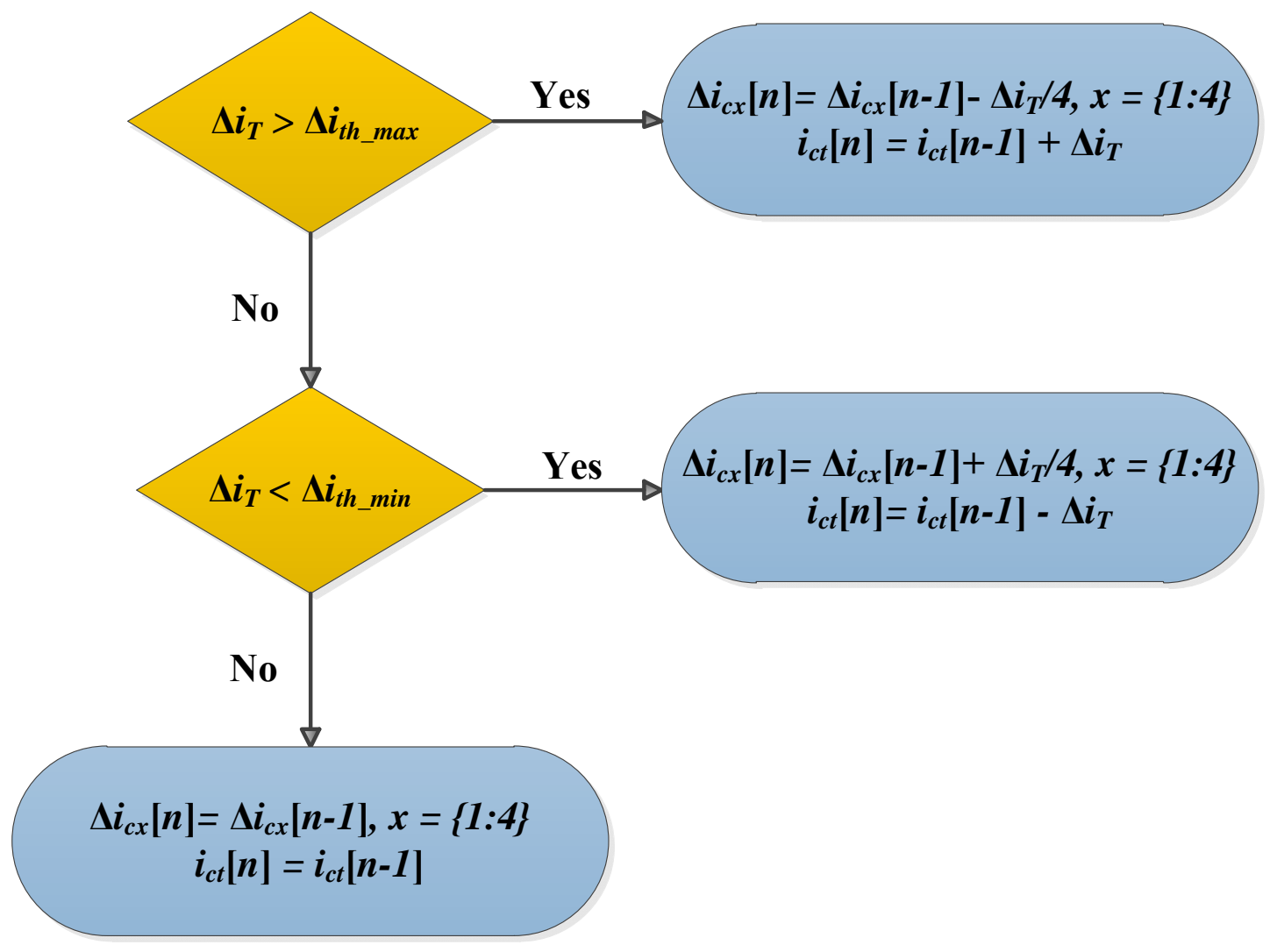

Figure 2.7: Offset block operation flow chart.

\subsection{MATLAB Simulation}

A mixed-signal simulation of the complete system in Figure 2.1 was performed using MATLAB Simulink to verify the TMU concept. The VRM specifications are shown in Table 2.1. The power electronic components are modeled using the SimPowerSystems toolbox. For each phase the average power losses over one switching cycle from the switches and inductor are fed into the thermal model of Figure 2.3. The average power losses are obtained by measuring the voltage across the devices and the current through the devices. Figure 2.8 and Figure 2.9 illustrate the implementation of the MATLAB Simulink model. 
Chapter 2. Design and Simulation of Multi-Phase Buck Converter with TMU

Table 2.1: VRM Specifications

\begin{tabular}{|l|c|c|}
\hline Parameter & Value & Unit \\
\hline Input voltage, $V_{\text {in }}$ & 12 & $\mathrm{~V}$ \\
\hline Output voltage, $V_{\text {out }}$ & 1 & $\mathrm{~V}$ \\
\hline Number of phases, $N$ & 4 & \\
\hline Inductance, $L$ & 0.68 & $\mu \mathrm{H}$ \\
\hline Total output capacitance, $C_{\text {out }}$ & 2 & $\mathrm{mF}$ \\
\hline Switching frequency, $f_{s}$ & 250 & $\mathrm{kHz}$ \\
\hline Maximum output current, $I_{\text {out }}$ & 50 & $\mathrm{~A}$ \\
\hline Nominal On-resistance for $M_{h s}, M_{l s}$ & 8 & $\mathrm{~m} \Omega$ \\
\hline
\end{tabular}

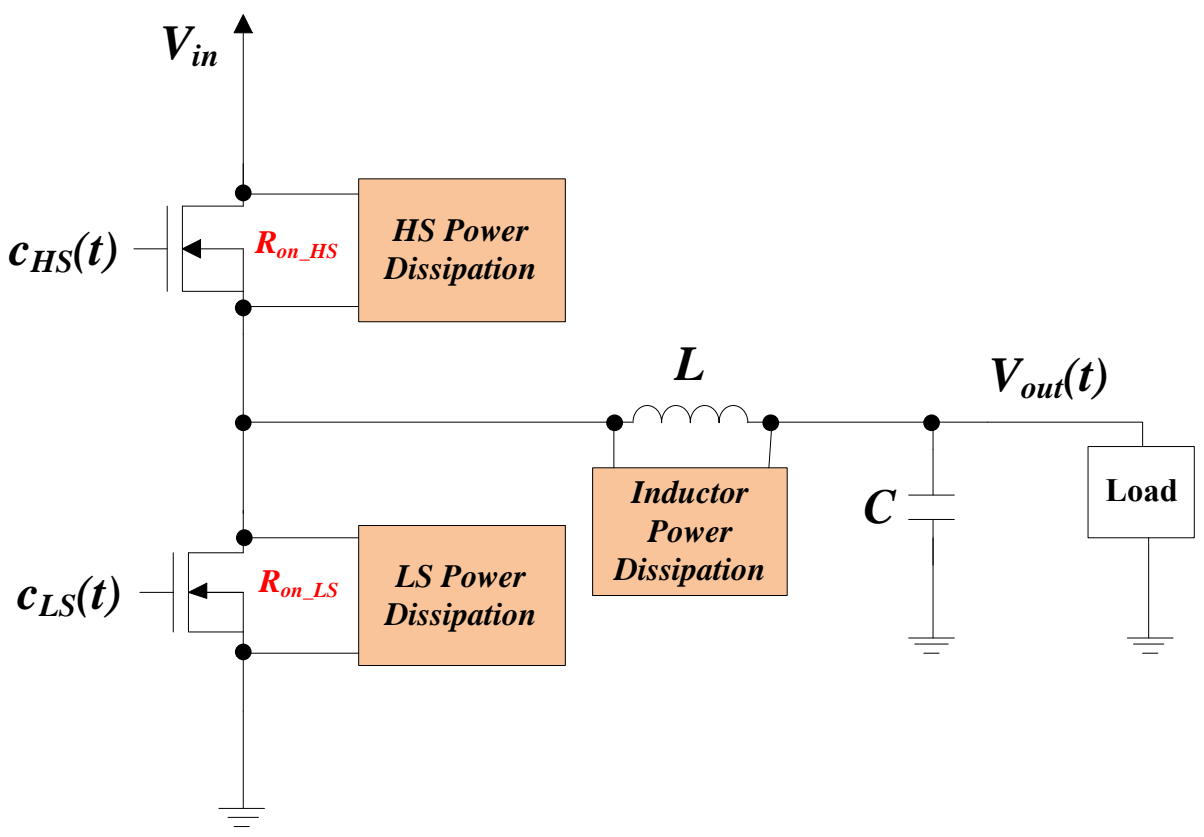

Figure 2.8: Power loss measurement of the switches and inductor for each phase. 
Chapter 2. Design and Simulation of Multi-Phase Buck Converter with TMU

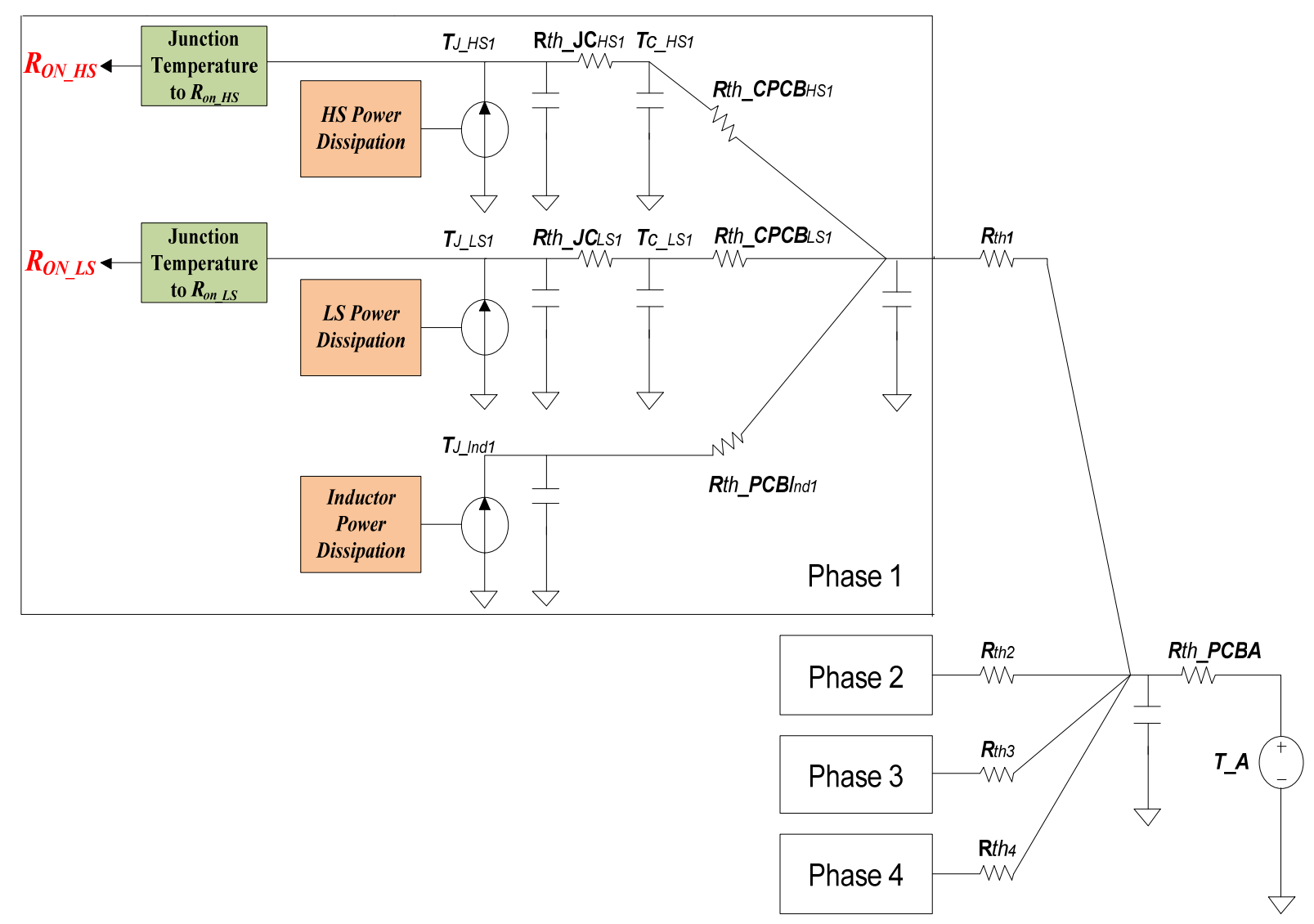

Figure 2.9: MATLAB thermal model.

The on resistance $\left(R_{O N}\right)$ of the MOSFET is temperature dependent as shown in Figure 2.10, which is a diagram taken from the FDD6782A N-Channel Power Trench ${ }^{\circledR}$ MOSFET datasheet. Therefore, the junction temperatures of the individual switches are also fed back into the electrical model to set the $R_{O N}$ according to Figure 2.10. The resulting setup provides a flexible platform for investigating the TMU operation. 
Chapter 2. Design and Simulation of Multi-Phase Buck Converter with TMU

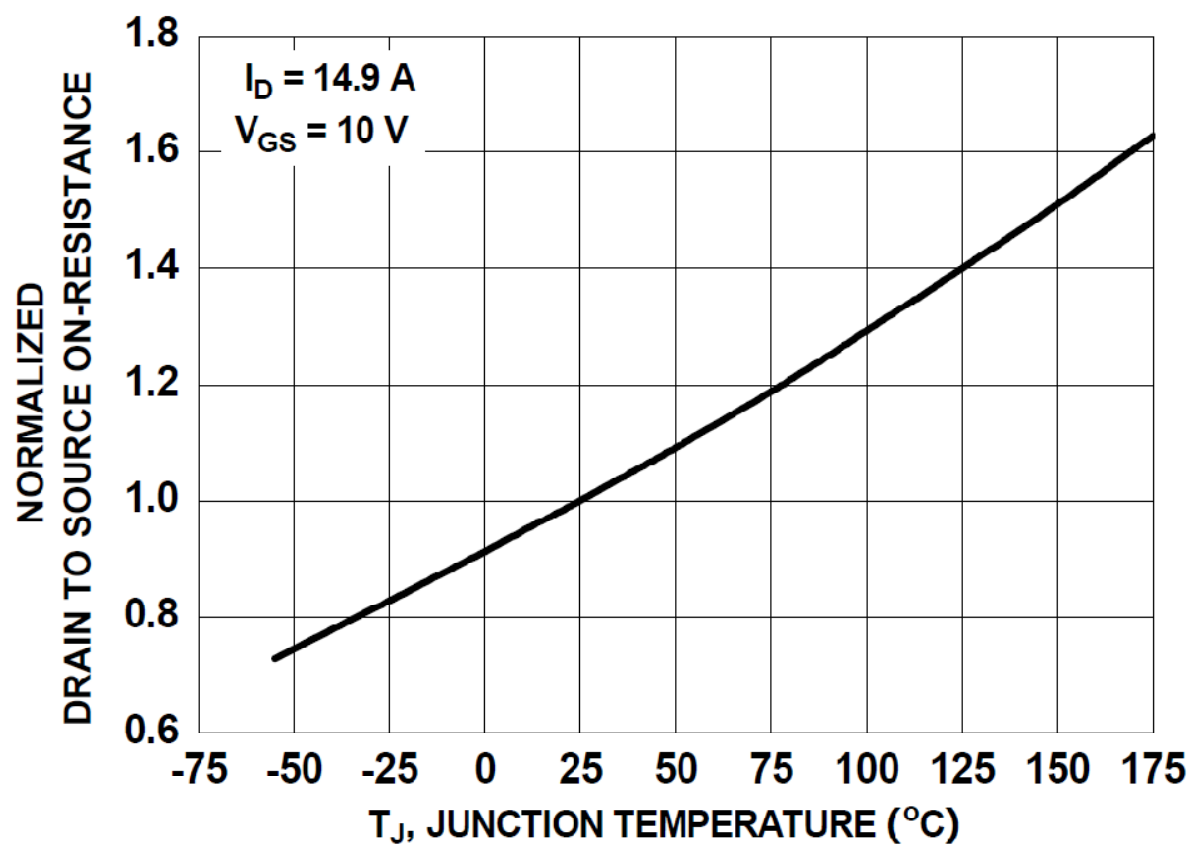

Figure 2.10: Normalized on-resistance vs. junction temperature [10].

All thermal capacitances can be scaled by a factor $K_{t}$ in order to accelerate the thermal settling time and reduce the simulation time. A $14 \mathrm{~ms}$ transient simulation took approximately 6 hours due to the complexity of the MATLAB circuit design without acceleration. To speed up the MATLAB Simulink simulation, the thermal capacitance is scaled down by about $1.3 \times 10^{5}$ times. The parameters in the thermal PI compensators are scaled accordingly. This large acceleration factor could cause oscillations when the voltage loop and thermal loop interact, such as when load step happens.

The simulation was performed for a $30 \mathrm{~A}$ to $50 \mathrm{~A}$ current load step at $t=7 \mathrm{~ms}$. The transient responses are as shown in Figure 2.11 and Figure 2.12 with the TMU disabled and enabled, respectively. 
Chapter 2. Design and Simulation of Multi-Phase Buck Converter with TMU
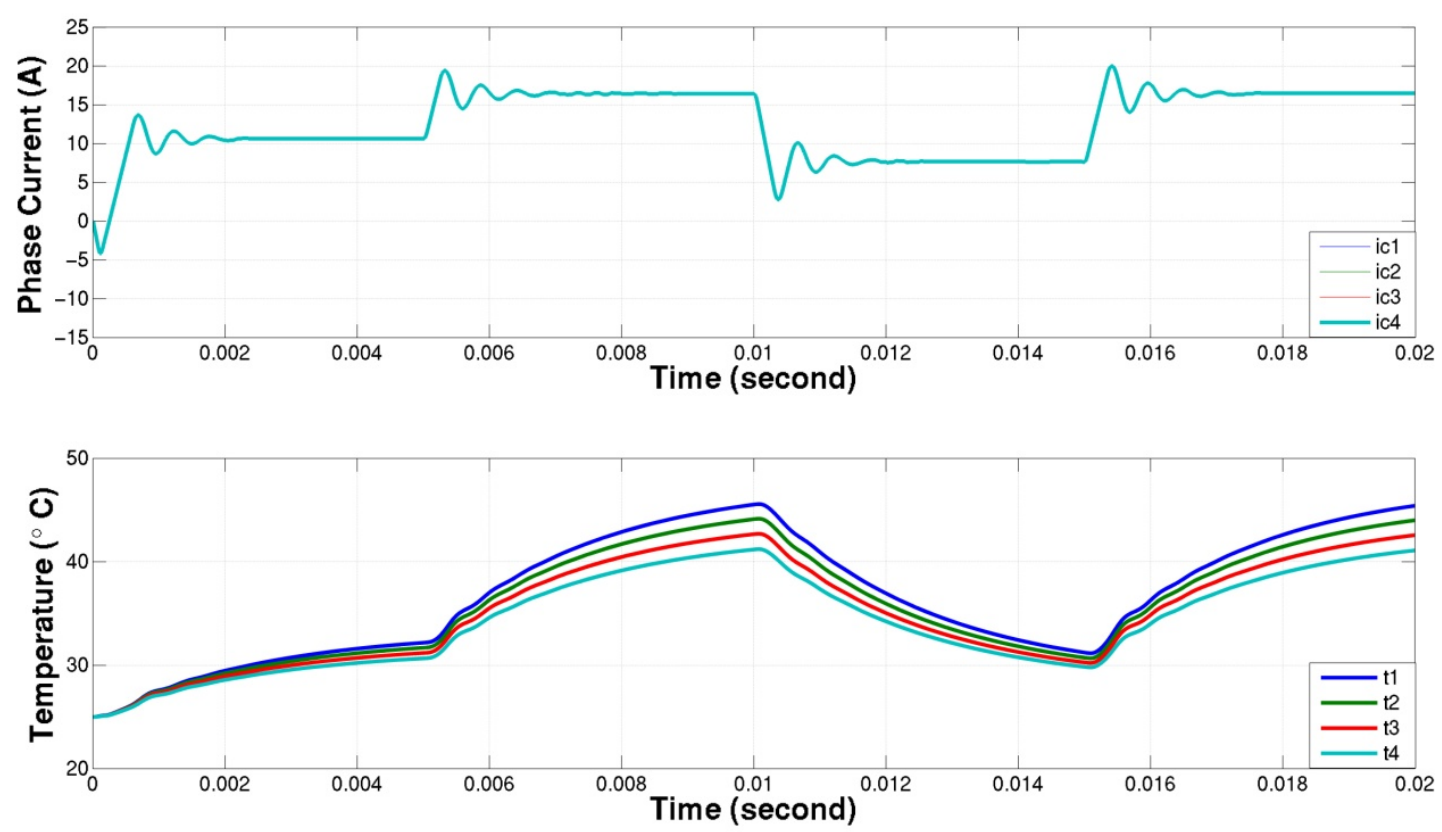

Figure 2.11: Simulated phase temperature with TMU disabled.
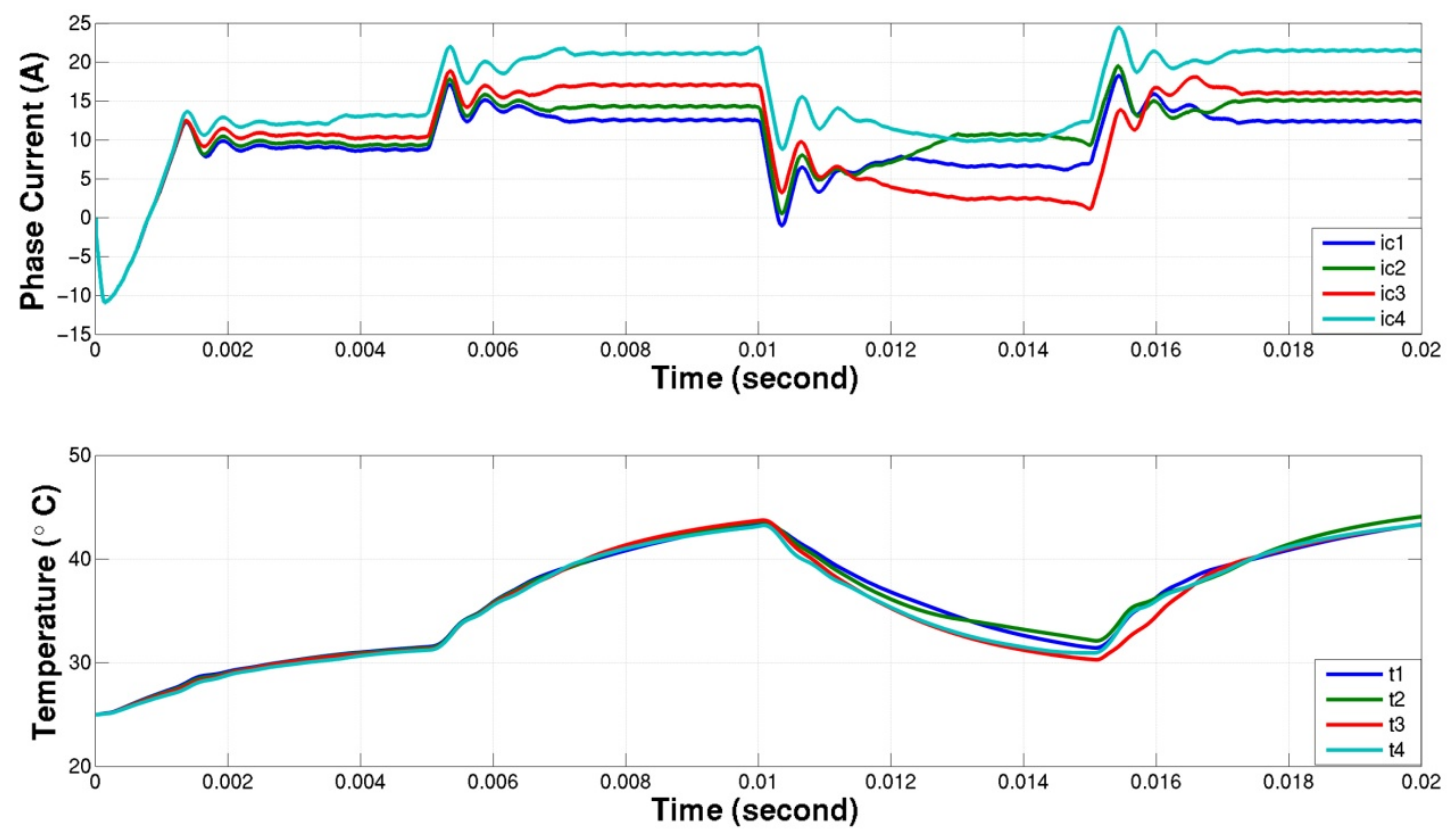

Figure 2.12: Simulated phase temperature with TMU enabled. 
The simulation results show that when the TMU was disabled, all the phases carry the same amount of current, but the phase temperatures spread out, as shown in Figure 2.11. When the TMU is enabled, the phase temperatures maintain a uniform distribution, while the phase currents are redistributed, as shown in Figure 2.12. Due to the large acceleration factor, there are slight oscillations in the response as the voltage and thermal loops interact.

The TMU concept is verified through the MATLAB Simulink simulations. The experimental results are presented and discussed in the following chapter to show the effectiveness of the TMU on a fabricated converter. 
Chapter 2. Design and Simulation of Multi-Phase Buck Converter with TMU

\section{References}

[1] Texas Instruments, “0.5 ${ }^{\circ} \mathrm{C}$ Digital Out Temperature Sensor,” Datasheet, August 2007.

[2] O. Trescases, “A High-Frequency, Soft-Switching DC-DC Converter for Dynamic Voltage Scaling in VLSI Loads," Master Thesis, 2004.

[3] C. Henze, H. Martin, and D. Parsley, "Zero-voltage switching in high frequency power converters using pulse width modulation, " in Proceedings, IEEE Power Electronics Specialists Conference, pp. 33-40, 1988.

[4] Q3D Extractor, Ansoft Inc., http://www.ansoft.com.

[5] S. Saggini and M. Ghioni, “An innovative digital control architecture for low-voltage highcurrent DC-DC converters with tight load regulation," IEEE Trans. Power Electron., vol. 19, no. 1, pp. 210-218, Jan. 2004.

[6] O. Trescases, Z. Lukic', W.-T. Ng, and A. Prodic', “A low power mixed-signal currentmode DC-DC converter using a one-bit delta sigma DAC," in Proc. IEEE Applied Power Electron. Conf. Expo., 2006, pp. 700-704.

[7] H. Peng and D. Maksimovic', "Digital current-mode controller for DC-DC converters," in Proc. IEEE Applied Power Electron. Conf. Expo., 2005, pp. 899-905.

[8] S. Chattopadhyay and S. Das, "A digital current-mode control technique for DC-DC converters," IEEE Trans. Power Electron., vol. 21, no. 6, pp. 1718-1726, 2006.

[9] Y.-S. Jung, "Small-signal model-based design of digital currrent-mode control," IEEE Proc. Elect. Power Appl., vol. 152, no. 4, 2005.

[10] "FDD6782A N-Channel PowerTrench MOSFET data sheet", Fairchild Semiconductor Corporation, Jan. 2009. 


\section{Chapter 3 Implementation and Experimental Results}

To verify the effectiveness of the TMU, a mixed-signal 4-phase CPM buck converter was fabricated and tested under non-uniform airflow and dynamic load transient conditions. The digital controller including the TMU was implemented using a FPGA platform. In this chapter, the physical implementation of TMU is introduced. Experiments were conducted to study the steady-state performance, dynamic transient performance, effect of gate drive voltage and efficiency.

\subsection{PCB Design}

A digitally-controlled 4-phase VRM with the specifications as given in Table 2.1 was built to verify the performance of the proposed TMU under load transients with non-uniform airflow. The electronic components selected to build the PCB are listed in Table 3.1. 
Chapter 3 - Implementation and Experimental Results

Table 3.1: Electronic Component Specifications and Comments

\begin{tabular}{|c|c|c|}
\hline Component & Part Number & Specifications and Comments \\
\hline N-Channel Power MOSFET & FDD6782A & $8 \mathrm{~m} \Omega$ nominal on-resistance \\
\hline Inductor & WE744331-0068 & 23 A rated current; $0.72 \mathrm{~m} \Omega \mathrm{DCR}$ \\
\hline Gate Driver & LM5100A & $30 \mathrm{~ns}$ rise time and $20 \mathrm{~ns}$ fall time \\
\hline $3.3 \mathrm{~V} \mathrm{LDO}$ & MIC29300-3.3BU & \\
\hline Linear Regulator & LT1129 & \\
\hline $\mathrm{DAC}$ & AD9740 & 10 bits resolution; $1.2 \mathrm{~V}$ reference \\
\hline $\mathrm{ADC}$ & AD9215 & 10 bits resolution; $1 \mathrm{~V}$ reference \\
\hline Comparator & TLV3501 & 4.5 ns rail-to-rail \\
\hline ADC buffer & AD8615 & Unity gain buffer; $20 \mathrm{MHz}$ bandwidth \\
\hline DAC and Comparator Buffer & LT1809 & Unity gain buffer; $320 \mathrm{MHz}$ bandwidth \\
\hline Temperature Sensor & TMP275 & $0.0625^{\circ} \mathrm{C}$ resolution; $0.5^{\circ} \mathrm{C}$ accuracy \\
\hline Current Sense Amplifier & AD8129 & Gain $=6 ; 200 \mathrm{MHz}$ bandwidth \\
\hline Voltage Reference & ZXRE060 & \\
\hline CPU Fan & EFB0412HНА & \\
\hline
\end{tabular}

The system block diagram of the mixed-signal 4-phase CPM buck converter is as shown in Figure 2.1. Figure 3.1 shows the fabricated converter. This PCB has 4 conductive layers, and it measures as 12.1 inch long and 5.9 inch wide. The PCB layout for the converter was designed to achieve symmetry between the four phases. The digital compensator and TMU are implemented on a Xilinx Virtex II Pro FPGA platform. A CPU fan was used to intentionally produce nonuniform airflow. 
The fan position and airflow in typical applications are optimized for the dominant source of heat, namely the CPU. The VRM cooling is a secondary consideration and so the phases are not necessarily symmetric with respect to the cooling. It should be noted that the airflow distribution used in this work is not necessarily typical for VRMs, but was used to intentionally generate a significant temperature differential in order to verify the TMU performance in extreme cases.

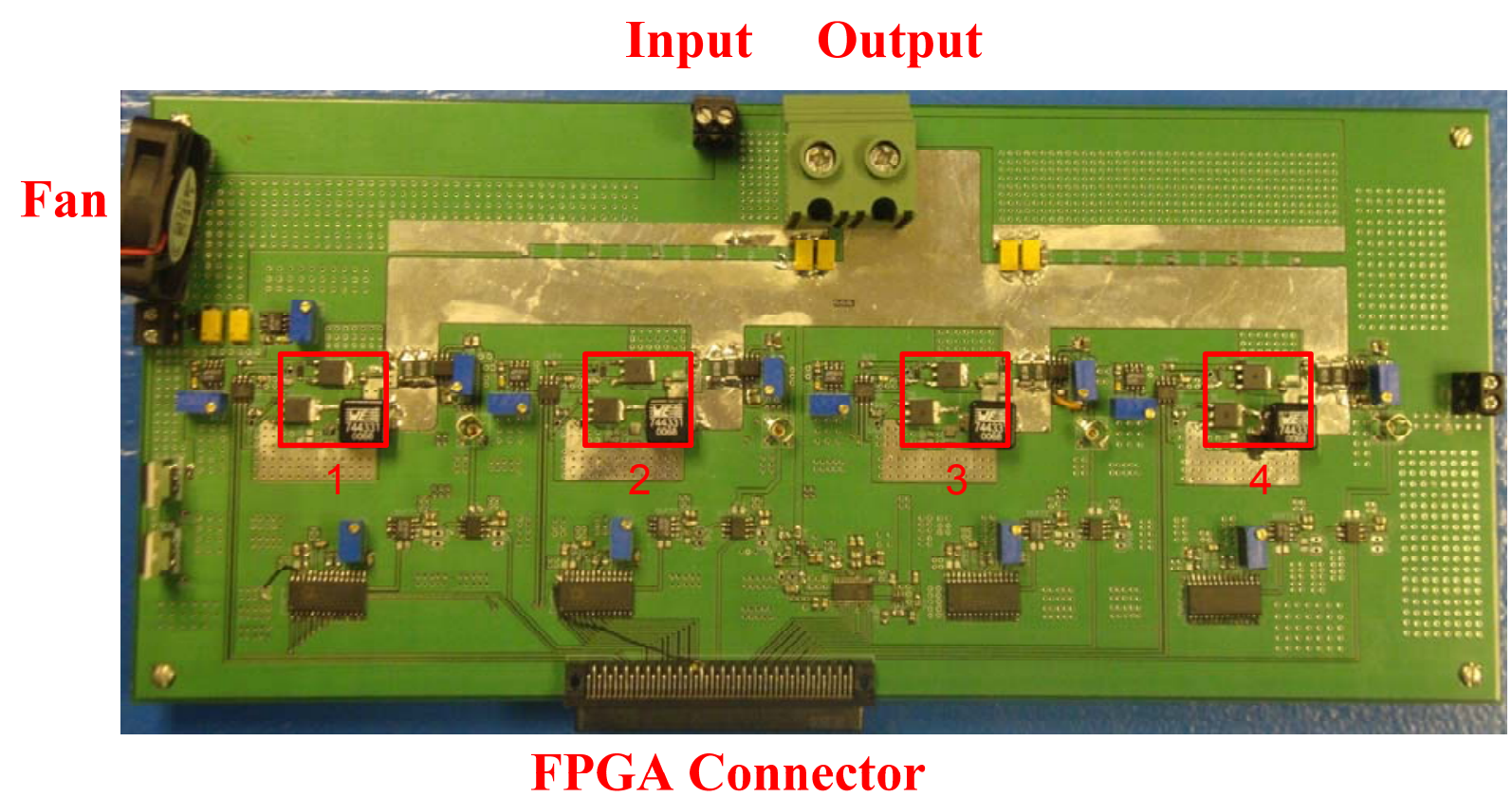

Figure 3.1: Fabricated mixed-signal 4 phase CPM buck converter.

The thermal parameter values in the lumped thermal model shown in Figure 2.3 are refined with this fabricated converter. The PCB materials and the length of the thermal paths directly affect the values of the thermal parameters. Due to the large area of the PCB, some thermal resistances are considered to be negligible, and Figure 2.3 shows the simplified thermal model of the system. The IR camera measures the temperature at different spots on the PCB. The thermal parameter values are calculated based on the temperature of these spots under current load steps. 


\subsection{Test Setup}

Two methods of introducing thermal mismatch were considered in this work. The gate-driver supply in each phase, $V_{d r v 1-4}$ is independently regulated to intentionally introduce on-resistance $\left(R_{O N}\right)$ variations according to the power MOSFET characteristics shown in Figure 3.2. The devices used in this work were chosen to have relatively high $R_{O N}(8 \mathrm{~m} \Omega$ nominal) in order to investigate the thermal behavior. Thermal mismatch is also introduced using a $12 \mathrm{~V}$ fan providing non-uniform airflow across the VRM, as discussed in the previous section.

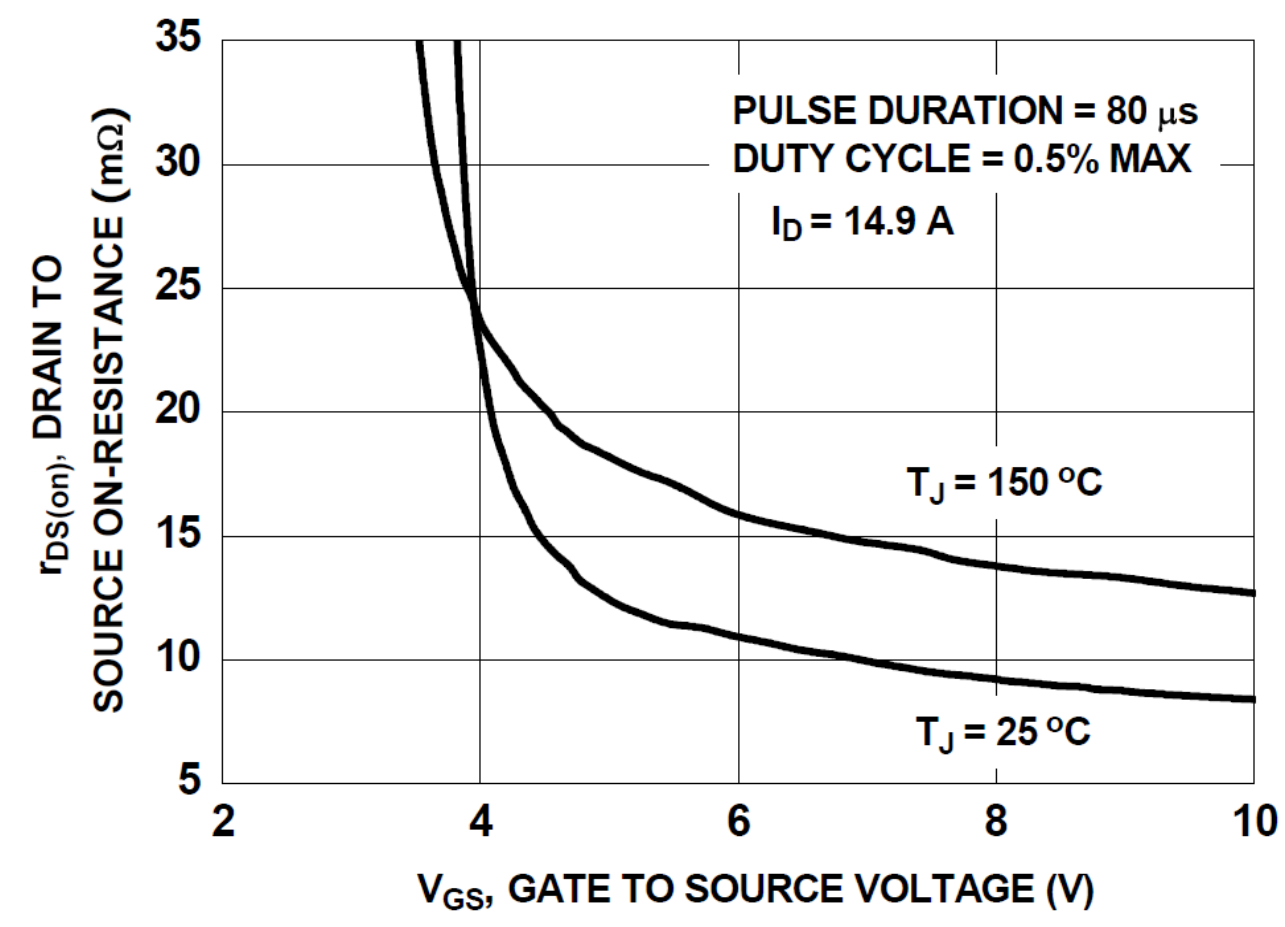

Figure 3.2: On-resistance of a power MOSFET as a function of gate to source voltage [1].

\subsection{Effect of Gate Drive Voltage}

The operation of the 4-phase converter was first tested with the fan turned off. The power-stage temperature for one of the phases is as shown in Figure 3.3 for varying total output current. The 
temperature was measured with two different gate drive voltages, $V_{d r v}$, to illustrate the effect of having different $R_{O N}$. As expected, the higher gate drive voltage generates less heat and is more efficient, since the MOSFET's $R_{O N}$ is inversely proportional to $V_{d r v}$.

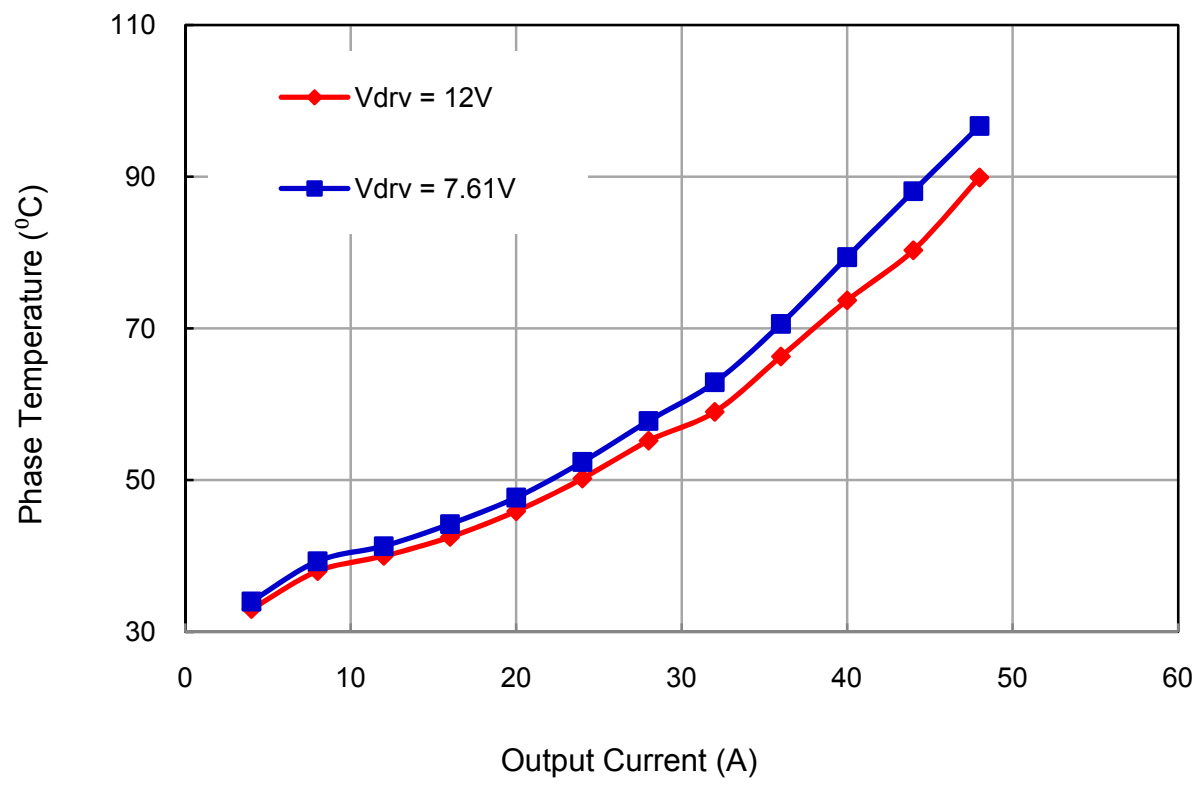

Figure 3.3: Measured temperature for one phase of the converter with the fan disabled.

\subsection{Power Efficiency Measurements}

Measured efficiency curves for the four operating conditions with the TMU and fan enabled or disabled are as shown in Figure 3.4. In all cases, the increased airflow results in a slightly higher efficiency as expected. The TMU has a minor impact on the efficiency. The TMU is expected to improve the reliability by reducing the temperature difference between different phases, and also reducing peak temperature of the entire system. This will be shown in subsequent tests. 


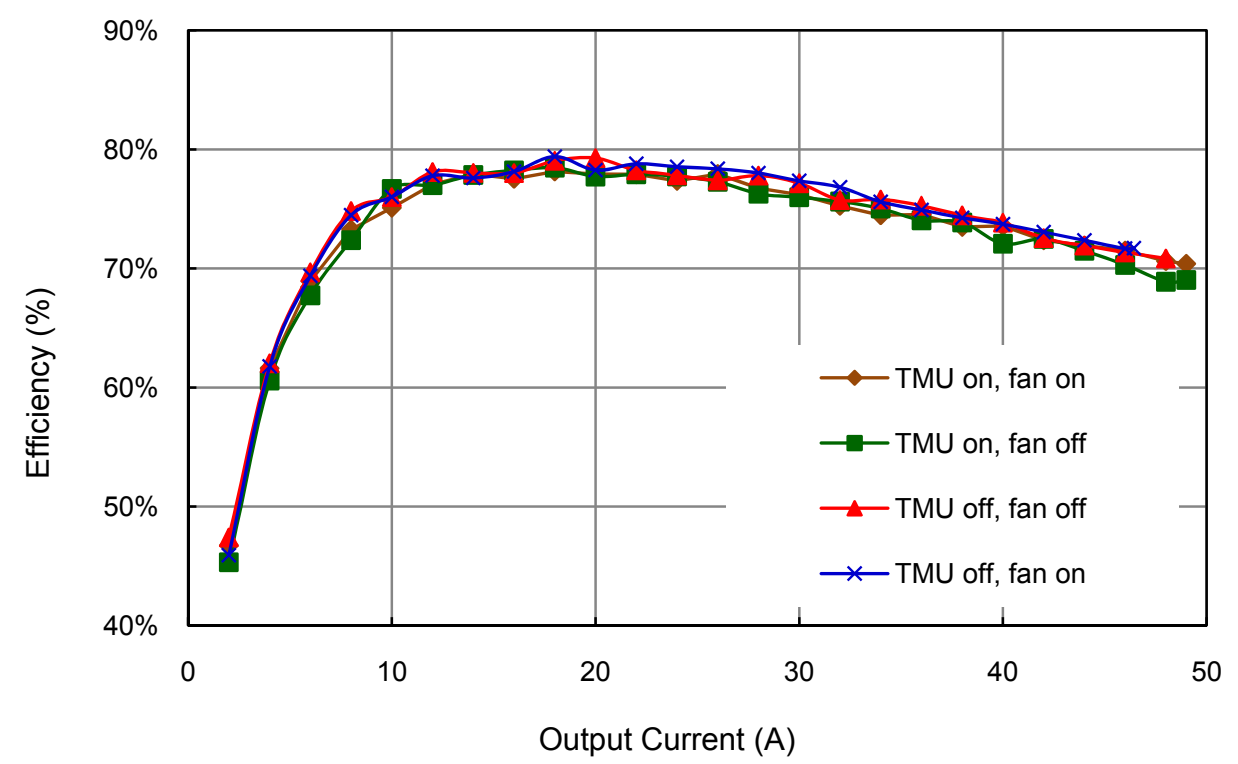

Figure 3.4: Measured efficiency for the four-phase converter under different operating conditions.

\subsection{Steady-State Performance}

Infrared (IR) images taken by a FLIR T300 camera were used to monitor the temperature distribution of the VRM with the TMU enabled and disabled, and under the presence of a nonuniform airflow. A thermographic camera or IR camera is a device that forms an image using infrared radiation. An IR image is a two dimensional temperature profile, which contains temperature data that can be read off by certain software. Usually the software can be obtained from the IR camera vendors.

The IR image with the TMU disabled is as shown in Figure 3.5, where all phases operate with an equal peak current command of $i_{c l-4}=i_{c t} / 4$. The fan was positioned parallel to the plane of the PCB with an airflow direction as indicated by the white arrow in Figure 3.5. The IR camera 
provides peak and average temperatures for the boxes defined around each power-stage, as shown in Figure 3.5.

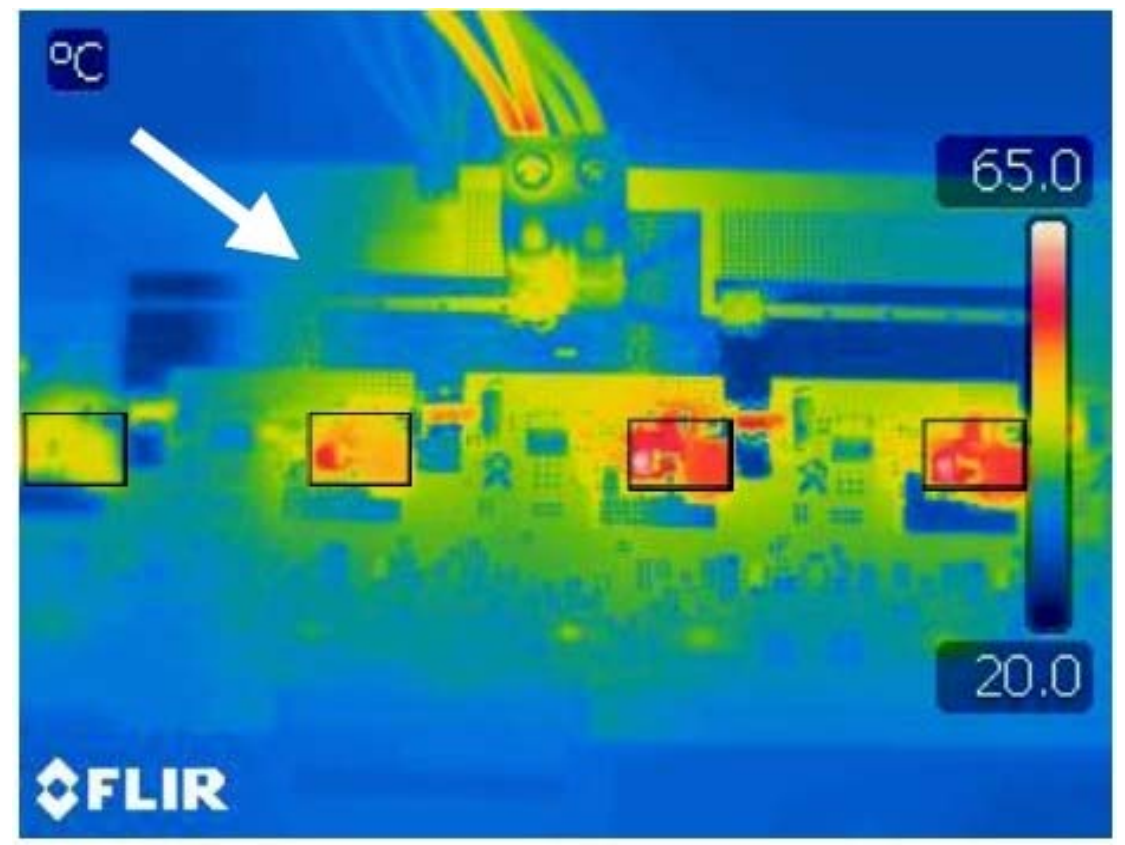

Figure 3.5: IR image with TMU disabled, and a total $I_{\text {out }}=40 \mathrm{~A}$. The arrow indicates the direction of airflow.

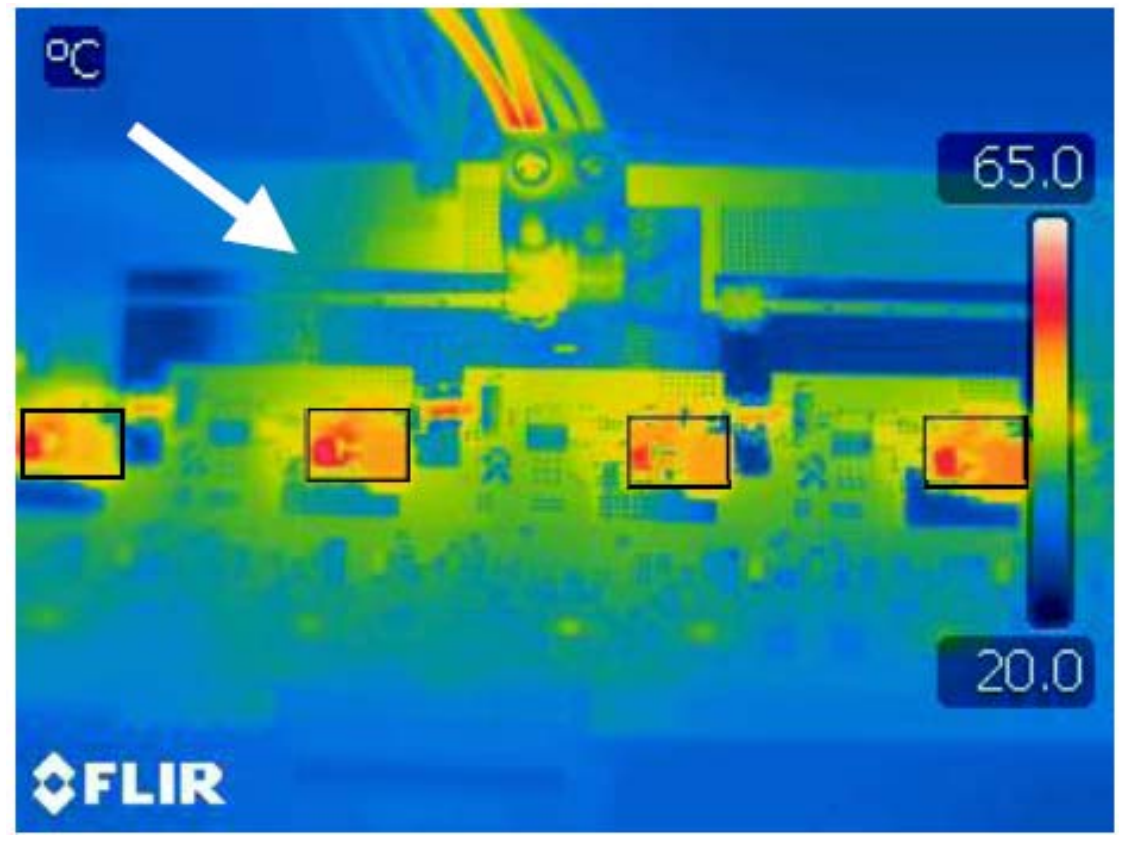

Figure 3.6: IR image with TMU enabled, with a total $I_{\text {out }}=40 \mathrm{~A}$. The arrow indicates the direction of airflow. 
The data in Table 3.2 indicates a maximum deviation of $8.9^{\circ} \mathrm{C}$ in average temperature between phase 1 and phase 3 when the TMU is off. This is mainly attributed to the non-uniform airflow, in addition to layout and parasitic component variations. A subsequent IR image taken with the TMU enabled is as shown in Figure 3.6. The maximum deviation in the average temperature between all phases is reduced from $8.9^{\circ} \mathrm{C}$ to $1.5^{\circ} \mathrm{C}$, and the peak phase temperature is reduced from $64.2^{\circ} \mathrm{C}$ to $60{ }^{\circ} \mathrm{C}$ within the power-stage area. This clearly shows the effectiveness of the TMU for achieving a uniform steady-state temperature distribution. The temperature data is measured independently from the embedded sensors using the IR camera, which confirms correct operation.

Table 3.2: Measured Temperature and Current Data for TMU Disabled and Enabled

\begin{tabular}{|c|c|c|c|c|c|c|c|c|}
\hline \multirow[b]{2}{*}{$\begin{array}{c}\text { Phase } \\
\text { No. }\end{array}$} & \multicolumn{4}{|c|}{ TMU off } & \multicolumn{4}{|c|}{ TMU on } \\
\hline & $\begin{array}{c}\text { Peak } \\
\text { Inductor } \\
\text { Current } \\
\text { (A) }\end{array}$ & $\begin{array}{c}\text { Average } \\
\text { Inductor } \\
\text { Current } \\
\text { (A) }\end{array}$ & $\begin{array}{c}\text { Peak } \\
\text { Temp. } \\
\left({ }^{\circ} \mathrm{C}\right)\end{array}$ & $\begin{array}{c}\text { Average } \\
\text { Temp. } \\
\left({ }^{\circ} \mathrm{C}\right)\end{array}$ & $\begin{array}{l}\text { Peak } \\
\text { Inductor } \\
\text { Current } \\
\text { (A) }\end{array}$ & $\begin{array}{c}\text { Average } \\
\text { Inductor } \\
\text { Current } \\
\text { (A) }\end{array}$ & $\begin{array}{c}\text { Peak } \\
\text { Temp. } \\
\left({ }^{\circ} \mathrm{C}\right)\end{array}$ & $\begin{array}{c}\text { Average } \\
\text { Temp. } \\
\left({ }^{\circ} \mathrm{C}\right)\end{array}$ \\
\hline 1 & 16.44 & 9.94 & 50.1 & 43.6 & 19.2 & 12.61 & 59.0 & 48.3 \\
\hline 2 & 16.53 & 10.13 & 57.4 & 48.9 & 17.24 & 10.50 & 60.0 & 49.8 \\
\hline 3 & 16.62 & 10.39 & 64.2 & 52.7 & 14.13 & 8.18 & 55.9 & 48.4 \\
\hline 4 & 17.16 & 10.10 & 62.6 & 52.5 & 16.44 & 9.12 & 58.1 & 49.7 \\
\hline
\end{tabular}

The steady-state average inductor currents are also measured as shown in Table 3.2. There is \pm $16 \%$ variation in the inductor phase currents when TMU is enabled. These current offsets regulated by the TMU contributed to minimizing the temperature difference between all phases. 
The fabricated converter in this work is about 5 to 6 times larger than the VRMs used for motherboards today. With the components more spread out, the phase temperature differences are much larger. Also, the non-uniform airflow was introduced to intentionally generate significant temperature differences to verify the effectiveness of the TMU under extreme cases. Therefore, large current offsets are observed with TMU enabled to equalize the phase temperatures. For the high power density commercial VRMs, the phase temperature differences are much smaller. Therefore, the current offsets are reduced accordingly, and no overrated components are required to compensate the current offsets. In addition, the components usually fail due to the high temperature. This thermal management technique is expected to improve the system reliability.

\subsection{Transient Performance of the TMU}

One of our main objectives of this work was to look beyond steady-state temperature equalization by investigating the transient response of the thermal system running in closed loop. In this section, the TMU performance was verified under transient load steps and realistic load current profile.

\subsubsection{Transient Load Steps}

Experiments with load steps were carried out to examine the dynamic transient performance of the TMU. The sensed temperature and peak current distributions for the multi-phase converter with the TMU disabled and enabled for a 30-50-20-50 A load step are as shown in Figure 3.7 and Figure 3.8, respectively. The TMU successfully maintains a uniform temperature distribution in 
all four phases, while the currents are re-distributed. The current offsets $\Delta i_{c 1-4}$ remains fixed immediately following a load step due to the low bandwidth of the thermal compensators. Feedforward from the current loop to the thermal loop could be added, however it does not seem necessary, given the fast response seen in Figure 3.8.

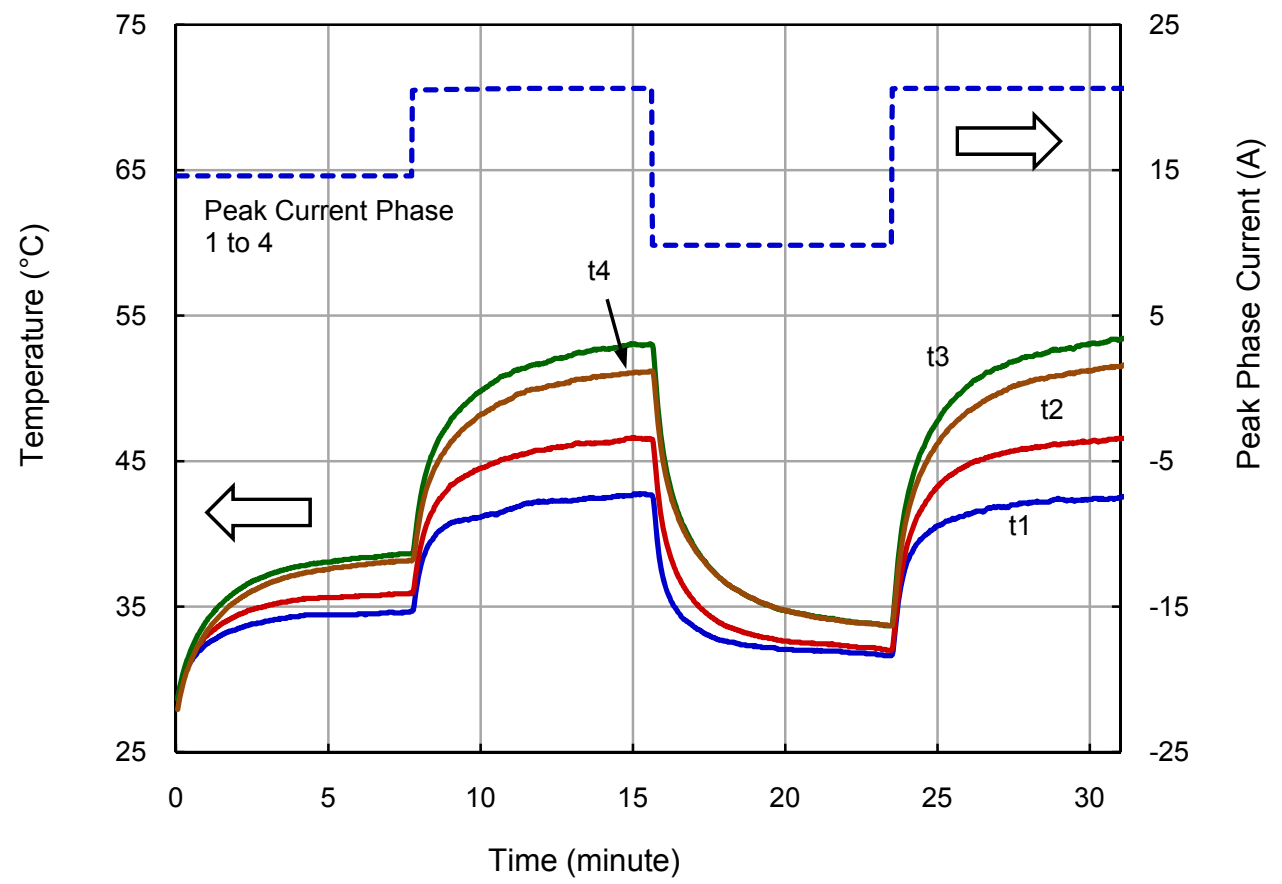

Figure 3.7: Temperatures measured using sensors mounted under each converter phase during a load transient with TMU disabled. 


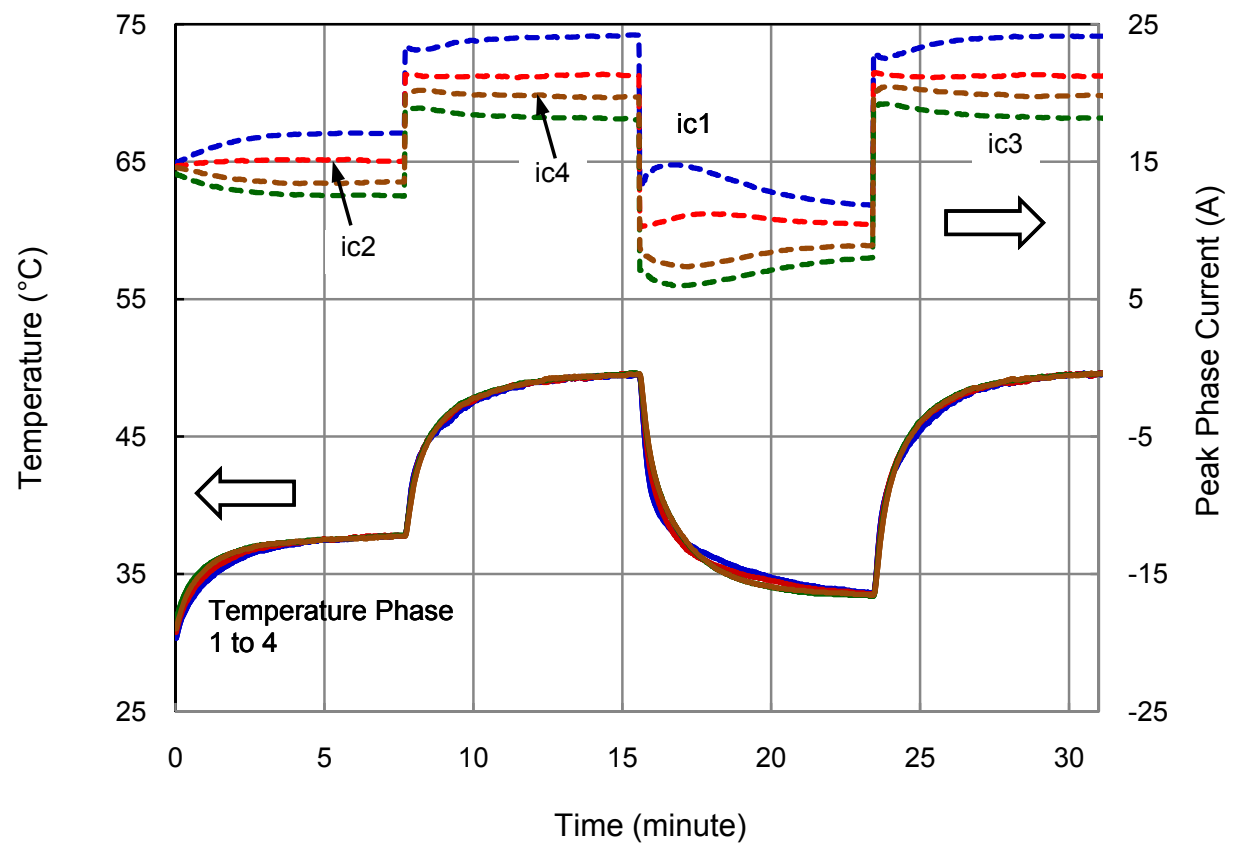

Figure 3.8: Temperatures measured using sensors mounted under each converter phase during a load transient with TMU enabled.

\subsubsection{Realistic Load Current Profile}

Another test was performed by subjecting the converter to a realistic current profile, obtained experimentally from a processor in a video decoding application. The high frequency and low frequency load current changes are superimposed. The current profile was programmed into the memory of an electronic load. The resulting thermal transient response is as shown in Figure 3.9 and Figure 3.11 with the TMU disabled and enabled, respectively. The temperature variations with TMU enabled and disabled are shown in Figure 3.10 and Figure 3.12. 
Chapter 3 - Implementation and Experimental Results

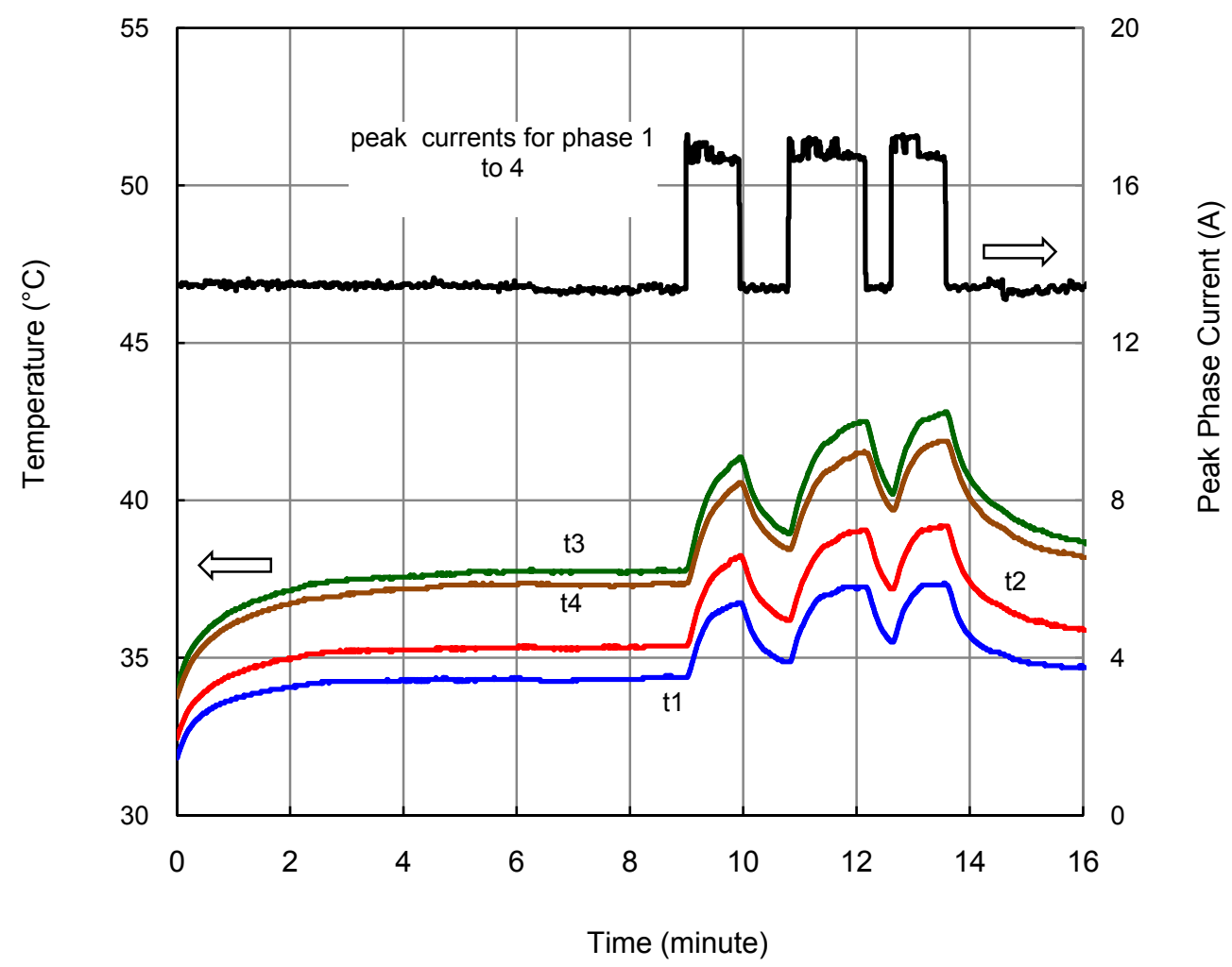

Figure 3.9: Temperatures measured during a realistic load current profile with TMU disabled.

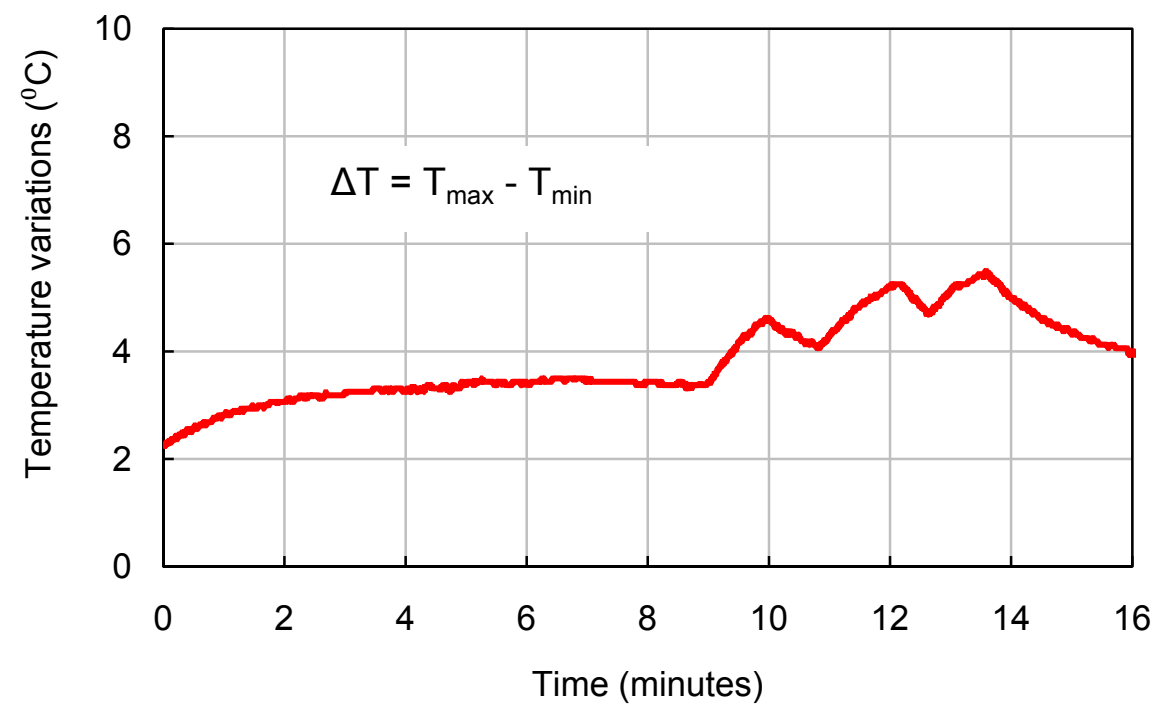

Figure 3.10: Temperature variations with TMU disabled. 
Chapter 3 - Implementation and Experimental Results

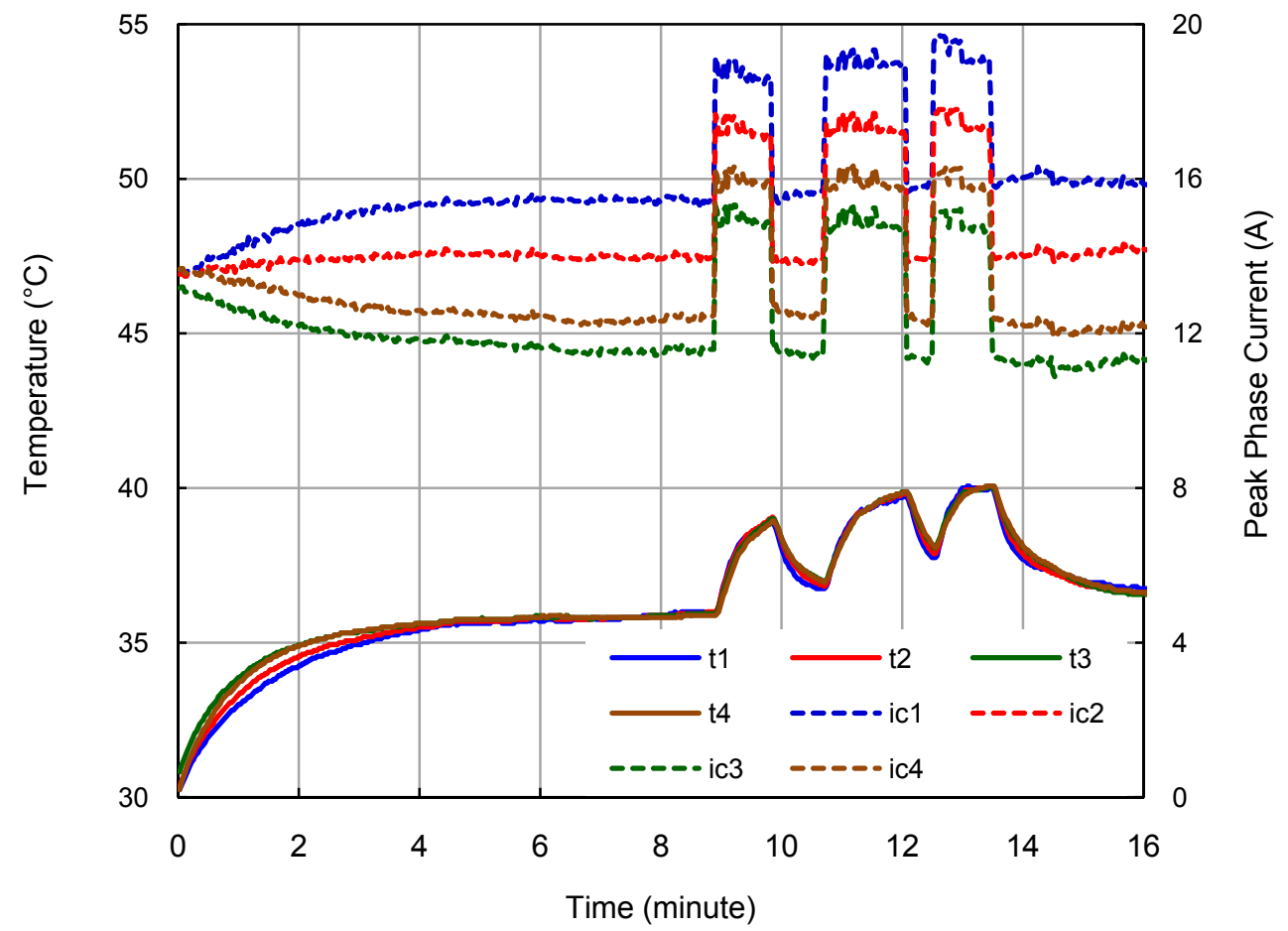

Figure 3.11: Temperatures measured during a realistic load current profile with TMU enabled.

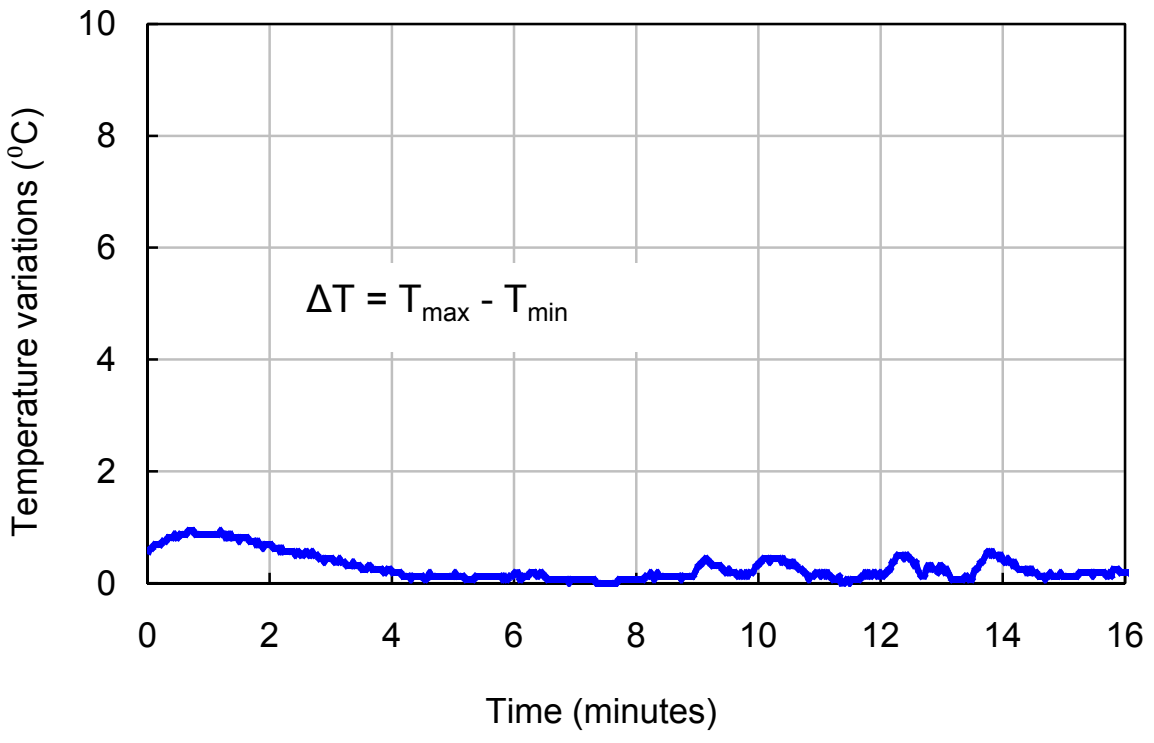

Figure 3.12: Temperature variations with TMU enabled. 


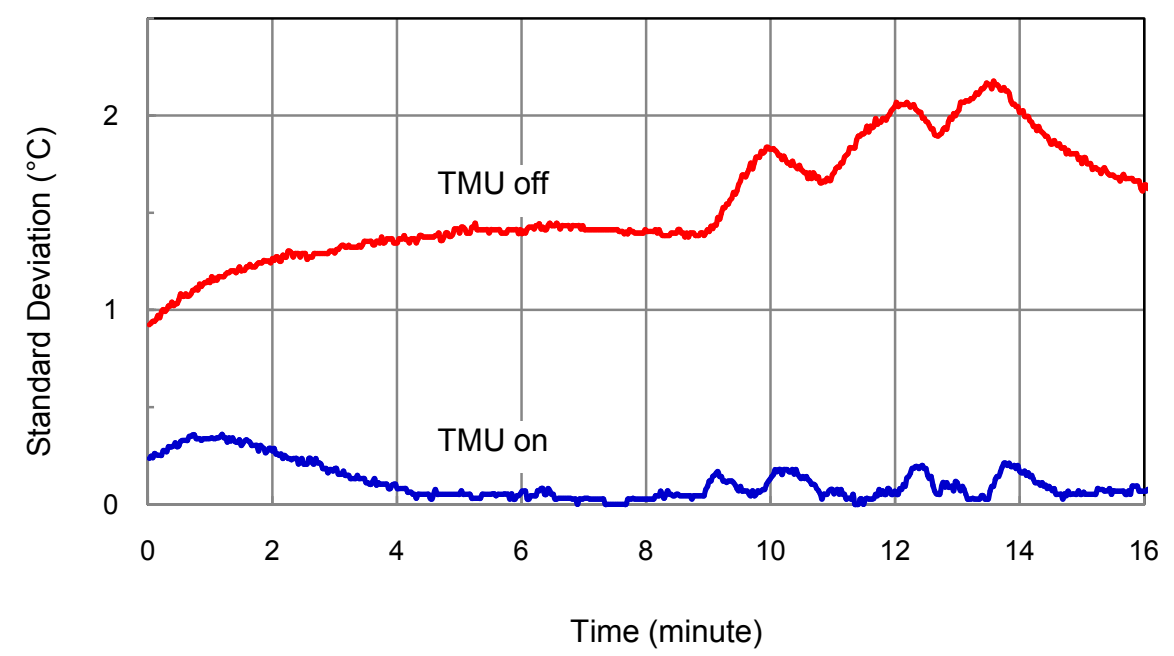

Figure 3.13: Standard deviation of the phase temperatures with TMU disabled and enabled.

With the TMU disabled, the maximum temperature difference between the hottest and coolest phases reached $5.5^{\circ} \mathrm{C}$. With the TMU enabled, there was barely any temperature difference except for the beginning when the system was just turned on. The TMU maintains a uniform temperature distribution even in the presence of realistic dynamic loads. The effectiveness of the TMU can also be observed from the measured standard deviation of the phase temperatures with TMU disabled and enabled as shown in Figure 3.13. It shows that the TMU actively tries to bring the standard deviation back to zero after every disturbance.

This digitally implemented TMU with dedicated PI compensators for the thermal loops is an innovative thermal management technique. This type of dynamic transient performance has not been shown in previous published work related to thermal management for multi-phase power converters.

In this chapter, the physical implementation of the TMU on a mixed-signal 4-phase CPM buck converter was introduced. Experiments were carried out under non-uniform airflow conditions. The effect of gate drive voltage was studied. The effectiveness of the TMU was verified through 
Chapter 3 - Implementation and Experimental Results

observing the steady-state and dynamic load transient performances. In conclusion, the TMU effectively regulated the current through each phase to achieve temperature equalization across all phases. This technique reduced the peak temperature of the PCB, and the maximum temperature between phases within the power stage area. Therefore, the MTBF and the long term reliability can be improved through this technique. 
Chapter 3 - Implementation and Experimental Results

\section{References}

[1] "FDD6782A N-Channel PowerTrench MOSFET data sheet", Fairchild Semiconductor Corporation, Jan. 2009. 


\section{Chapter 4 Conclusions and Future Work}

In this chapter, the design, simulation and testing results of the proposed thermal mangement technique are briefly summarized. Potential ideas for future work are introduced.

\subsection{Thesis Summary}

In conventional designs, precise current sharing has been used for multi-phase VRMs to reduce the voltage and current ripple, while presumably minimizing thermal stress. However, due to variations in PCB layout, parasitic resistances, airflow, and semiconductor parameters, significant temperature variations are observed between the converter phases, which leads to reliability issues and sub-optimal device utilization. In this work, an active thermal management control technique for multi-phase peak current mode controlled VRMs was introduced. A thermal management unit (TMU) was incorportated into the existing digital controller to regulate the current through each phase in order to equalize the temperature across all phases.

The TMU was designed with independent linear PI compensators for the thermal loops. A simple lumped-parameter thermal model of the VRM was developed to serve as the basis for the TMU design. MATLAB Simulink was used to verify the TMU concept. To further evaluate the effectiveness of this thermal management technique, a $12 \mathrm{~V}$ to $1 \mathrm{~V}, 50 \mathrm{~A}, 250 \mathrm{kHz}$, four-phase mixed-signal CPM buck converter was fabricated. The digital controller including the TMU was implemented using an FPGA platform.

The TMU was evaluated from several aspects, which include steady-state performance, dynamic transient performance, and efficiency measurements. The effect of gate drive voltage was also 
studied. Non-uniform airflow distribution was used to create significant temperature difference between phases and to test the TMU under extreme cases.

One of the main objectives of this work was to look beyond the steady-state temperature equalization by investigating the dynamic transient response of the thermal system running in closed loop. The IR images and on-board temperature measurements confirm the effectiveness of the TMU even under rapid load transient. Good performance was achieved despite limitations in the thermal model accuracy. The ability to equalize the temperature across different converter phases ensures greater reliability. This work was presented at the Applied Power Electronics Conference 2011 [1].

\subsection{Future Work}

Research in thermal management for power converters is an on-going process. In relation to this thesis, below are some suggestions for future work and some topics for further exploration.

Multivariable TMU Controller Design: The independent PI compensator TMU design in this work lacks of communication between all phases. Therefore, an offset control block is required to prevent the registers from overflowing, as the offset current command and the central current command from the voltage loop grow in opposite directions. Multivariable control considers the temperature and current information from all phases, and the offset control block could be eliminated. Mohammad Shawkat Zaman (M.A.Sc. student) has been working on the same topic with $H^{2}$ Optimization multivariable control technique. 
Extraction of Thermal Model Parameters: To model the behaviour of the entire system more accurately, the extraction of the thermal model parameters could be improved. A linear superposition technique could be investigated to extract the thermal resistances.

Temperature Sensor Implementations: The effect of different temperature sensor positions on the TMU performance could be evaluated. Different temperature sensor implementation methods could be considered as well. Since the TMU operation is based on the temperature sensor readings, the optimal positions for the temperature sensors are where dominant power loss sources are. In this way, the temperature equalization effect is optimized, and the peak temperature of the entire system is reduced most effectively.

Airflow Scenarios: Different airflow scenarios could be studied to examine the TMU performance under extreme cases. In addition, the combination of different PCB orientations and fan positions could cause the thermal model to be different.

Variable Speed Fan Control: Incorporating the TMU technique into variable fan speed control could be a potential application. Nowadays, most motherboards control the fan speed based on the CPU temperature. VRM cooling is the secondary consideration. However, with increase of the amount of the current the VRM need to supply, the VRM temperature should be taken into consideration for fan speed control. The TMU is able to reduce the peak temperature of the VRM system. The temperature information accquired by the TMU can also be used to help decide on the fan speed. 
Chapter 4 - Conclusions and Future Work

\section{References}

[1] K. Cao, W. T. Ng, and O. Trescases, "Thermal Management for Multi- Phase Current Mode Buck Converters," in Proc. APEC 2011, (accepted for publication), March 2011 


\section{Appendix A Small-Signal AC Model of CPM Buck Converter}

Suppose that the converter is driven at some steady-state, or quiescent, duty ratio $d(t)=D$, with quiescent input voltage $v_{g}(t)=V_{g}$. The steady-state analysis can be performed with the principle of inductor volt-second balance and the principle of capacitor charge balance. They are stated as (A1) and (A2)

$$
\begin{aligned}
& 0=\frac{1}{T} \int_{0}^{T_{S}} v_{L}(t) d t \\
& 0=\frac{1}{T} \int_{0}^{T_{S}} i_{C}(t) d t
\end{aligned}
$$

where

$$
\begin{aligned}
& v_{L}(t)=L \frac{d i_{L}(t)}{d t} \\
& i_{C}(t)=C \frac{d i_{L}(t)}{d t} .
\end{aligned}
$$

After any transients have subsided, the inductor current $\langle i(t)\rangle_{T s}$, the capacitor voltage $\langle v(t)\rangle_{T s}$, and the input current $\left\langle i_{g}(t)>_{T s}\right.$ will reach the quiescent values $I, V$, and $I_{g}$, respectively, where

$$
\begin{aligned}
& V=D V_{g} \\
& I=\frac{V}{R} \\
& I_{g}=D I
\end{aligned}
$$

To construct a small-signal ac model at a quiescent operating point $(I, V)$, one assumes that the input voltage, the duty cycle, the averaged inductor current, the averaged capacitor voltage, and 
the averaged input current are equal to some given quiescent values $V_{g}, D, I, V$, and $I_{g}$, plus some superimposed small ac variations $\hat{v}_{\mathrm{g}}(\mathrm{t}), \hat{\mathrm{d}}(\mathrm{t}), \hat{\imath}(\mathrm{t}), \hat{v}(\mathrm{t}), \hat{l}_{\mathrm{g}}(\mathrm{t})$. With the assumption that the ac variations are small in magnitude compared to the dc quiescent values, the nonlinear equations can be linearized. The second-order ac terms are much smaller in magnitude than the first-order ac terms, so the second-order ac terms are neglected. The dc terms on the right hand side of the equation are equal to the dc terms on the left-hand side, or zero. The rest are the first-order ac terms on both sides of the equation

$$
\begin{gathered}
L \frac{d \hat{\imath}(t)}{d t}=\hat{d}(t) V_{g}+D \hat{v}_{g}(t)-\hat{v}(t) \\
C \frac{d \hat{v}(t)}{d t}=\hat{\imath}(t)-\frac{\hat{v}(t)}{R} \\
\hat{\imath}_{g}(t)=D \hat{\imath}(t)-I \hat{d}(t) .
\end{gathered}
$$

Based on (A8) - (A10), the averaged small-signal ac model for buck converter operating in continuous conduction mode (CCM) can be constructed as shown in Figure A.1. This is an equivalent circuit that models the low-frequency small-signal variations in the converter waveforms. It can now be solved, using conventional linear circuit analysis techniques, to find the converter transfer functions, output impedance, and other ac quantities of interest.

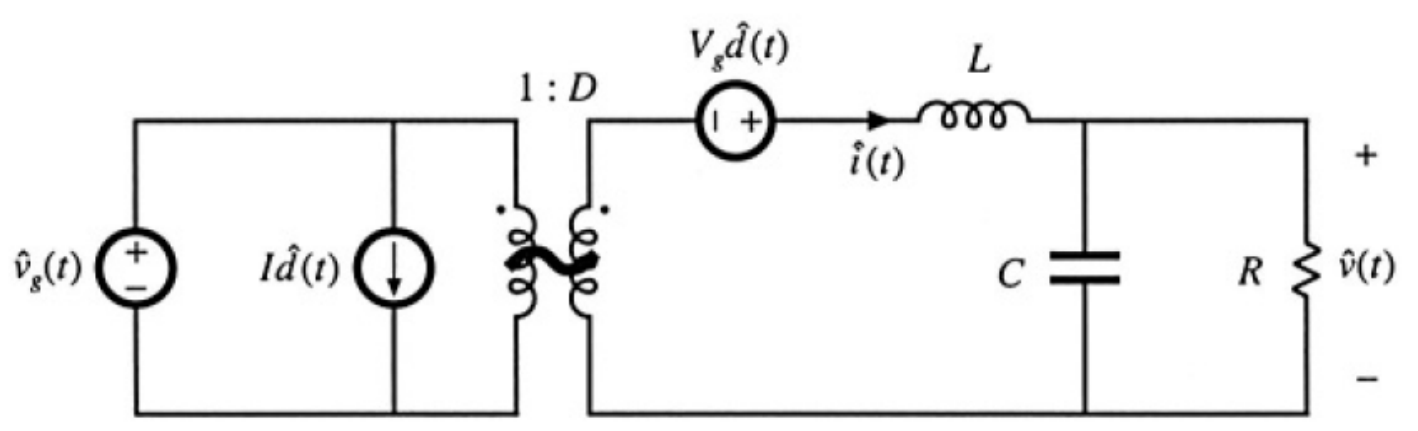

Figure A.1: Averaged small-signal ac models for buck converter operating in CCM [1]. 
With initial conditions set to zero, the Laplace transforms of (A8) - (A10) are performed:

$$
\begin{gathered}
s L \hat{\imath}_{L}(s)=V_{g} \hat{d}(s)+D \hat{v}_{g}(s)-\hat{v}(s) \\
s C \hat{v}(s)=\hat{\imath}_{L}(s)-\frac{\hat{v}(s)}{R} \\
\hat{\imath}_{g}(s)=D \hat{\imath}_{L}(s)+I_{L} \hat{d}(s)
\end{gathered}
$$

For the current programmed mode control, it is assumed that the inductor current $\hat{l}_{L}(s)$ is identical to the programmed control current $\hat{t}_{c}(s)$. This is valid to the extent that the controller is stable, and the magnitudes of the inductor current and artificial ramp waveforms are sufficiently small:

$$
\hat{\imath}_{L}(s) \approx \hat{\imath}_{c}(s)
$$

With this approximation, in conjunction with the inductor current equation of (A11), the relationship between the control current $\hat{l}_{c}(s)$ and the duty cycle $\widehat{\mathrm{d}}(\mathrm{s})$ can be found. By substituting this relationship into (A12) and (A13), $\widehat{\mathrm{d}}(\mathrm{s})$ can be eliminated. One obtains

$$
\begin{gathered}
s C \hat{v}(\mathrm{~s})=\hat{\imath}_{c}(s)-\frac{1}{R} \hat{v}(\mathrm{~s}) \\
\hat{\imath}_{g}(s)=\left(D+\frac{s L D}{R}\right) \hat{\imath}_{c}(s)+\frac{D}{R} \hat{v}(\mathrm{~s})-\frac{D^{2}}{R} \hat{v}_{g}(s)
\end{gathered}
$$




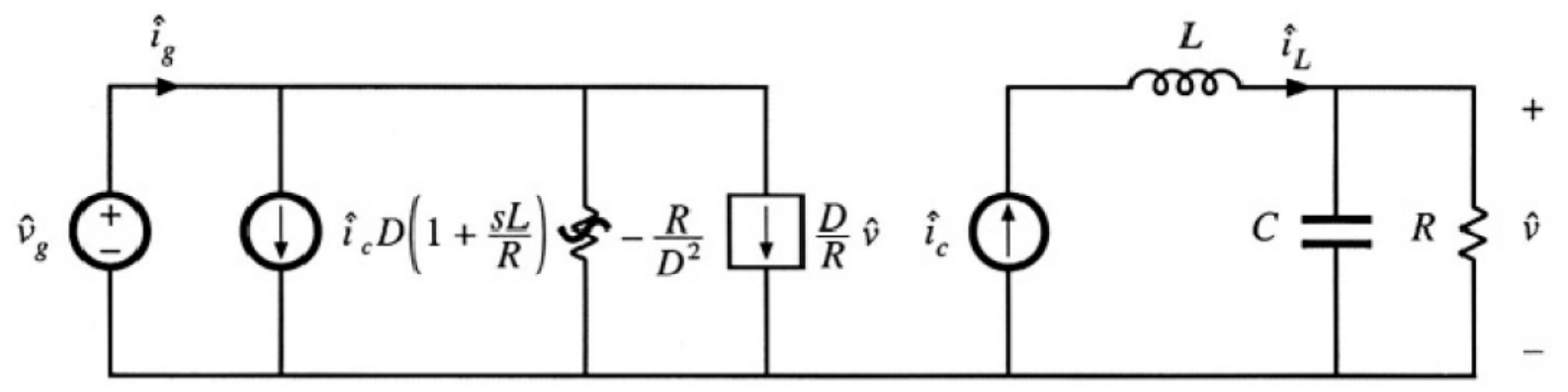

Figure A.2: Small-signal model of the CCM CPM buck converter [1].

Based on (A15) and (A16), the small-signal two-port model of Figure A.2 is constructed. The converter transfer functions and output impedance can be found by solving the two-port equivalent circuit. The control-to-output transfer function is determined by setting $v_{g}$ to zero:

$$
G_{v c}(s)=\left.\frac{\hat{v}(s)}{\hat{\imath}_{C}(s)}\right|_{\hat{v}_{g}=0}=\left(R \| \frac{1}{s C}\right)=\frac{R}{1+s C R}
$$

It can be seen that this transfer function contains only one pole and this system can be compensated with a PI controller.

Figure A.1 predicts that the buck converter line-to-output transfer function is zero:

$$
G_{v g}(s)=\left.\frac{\hat{v}(s)}{\hat{v}_{g}(s)}\right|_{\hat{\imath}_{c}=0}=0
$$

Disturbances in $v_{g}$ do not influence the output voltage, since the inductor current depends only on $i_{c}$. The current programmed controller adjusts the duty cycle as necessary to maintain constant inductor current, regardless of variations in $v_{g}$. 


\section{References}

[1] R. Erickson and D. Maksimovic, Fundamentals of Power Electronics. Norwell, MA: Kluwer, 2001. 


\section{Appendix B Thermal Parameter Values Extracted for the Experimental Prototype}

Table B.1: Thermal Resistance Values Extracted for the Experimental Prototype

\begin{tabular}{|c|c|}
\hline Thermal Parameters & Values $(\mathbf{K} / \mathbf{W})$ \\
\hline$R_{t h \_} J C_{H S 1-4}$ & 2.7 \\
\hline$R_{t h \_} J C_{L S 1-4}$ & 2.7 \\
\hline$R t_{h_{-}} C P C B_{H S 1-4}$ & 15.38 \\
\hline$R_{t h \_} C P C B_{L S 1-4}$ & 2.94 \\
\hline$R_{t h \_} P C B_{\text {Indl-4 }}$ & 3.47 \\
\hline$R_{t h l-4}$ & 0.68 \\
\hline$R_{t h \_} P C B A$ & 0.28 \\
\hline
\end{tabular}

Table B.2: Thermal Capacitance Values Extracted for the Experimental Prototype

\begin{tabular}{|c|c|}
\hline Thermal Capacitance & Values $(\mathbf{J} / \mathbf{K})$ \\
\hline$C_{t h \_} J C_{H S I-4}$ & 541.62 \\
\hline$C_{t h \_} J C_{L S I-4}$ & 491.53 \\
\hline$C_{t h \_} P S$ & 1653.6 \\
\hline$C_{t h \_} P C B$ & 5788.27 \\
\hline
\end{tabular}

